Annual Review of Earth and Planetary Sciences, In Press

\title{
The compositions of Kuiper belt objects
}

\author{
Michael E. Brown \\ Division of Geological and Planetary Science, California Institute of Technology, Pasadena, CA \\ 91125
}

\begin{abstract}
Objects in the Kuiper belt are small and far away thus difficult to study in detail even with the best telescopes available at earth. For much of the early history of the Kuiper belt, studies of the compositions of these objects were relegated to collections of moderate quality spectral and photometric data that remained difficult to interpret. Much early effort was put into simple correlations of surface colors and identifications of spectral features, but it was difficult to connect the observations to a larger understanding of the region. The last decade, however, has seen a blossoming in our understanding of the compositions of objects in the Kuiper belt. This blossoming is a product of the discoveries of larger - and thus easier to study - objects, continued dedication to the collection of a now quite large collection of high quality photometric and spectroscopic observations, and continued work at the laboratory and theoretical level. Today we now know of many processes which affect the surface compositions of objects in the Kuiper belt, including atmospheric loss, differentiation and cryovolcanism, radiation processing, the effects of giant impacts, and the early dynamical excitation of the Kuiper belt. We review the large quantity of data now available and attempt to build a comprehensive framework for understanding the surface compositions and their causes. In contrast to surface compositions, the bulk compositions of objects in the Kuiper belt remain poorly measured and even more poorly understood, but prospects for a deeper understanding of the formation of the the outer solar are even greater from this subject.
\end{abstract}

\section{Introduction}

The region of the solar system beyond the planets is populated by a vast swarm of objects in what is called the Kuiper belt. The objects in this swarm - Kuiper belt objects (KBOs) - formed on the outer edge of the planetary system where nebular densities were too low and accretional timescale too high to allow objects to accumulate into a single dominant planet, as happened throughout the planetary system. Instead, in the region beyond the planets, accretion proceeded up to objects at least as large as the $2700 \mathrm{~km}$ diameter of Triton but then stalled as dynamical 
excitation of the region caused collision velocities to be too high to allow continued accretion. Today, encounters between objects in the outer solar system lead to erosion to smaller diameters.

Much of we have learned from the Kuiper belt has come from study of the dynamics of these bodies (i.e. Morbidelli, Levison \& Gomes 2008). Understanding the dynamical state of a KBO is straightforward: after an object is discovered, a few observations spaced over several years are usually sufficient to define a precise orbit for the object (Bernstein \& Khushalani 2000). Since their formation KBOs have been moved around by the gravitational tugs and perturbations of the giant planets, and their current locations preserve a record of these billions of years of interactions. Our current understanding of the rearrangement and migration of the giant planets is based largely on this record.

In principle, the objects in the Kuiper belt also contain a wealth of information on the chemical conditions in the earliest solar system. The materials in these objects have, for example, avoided much of the high temperature and pressure processing to which materials in the inner solar system have been subjected. In practice, however, understanding the detailed composition of KBOs in their native environment has proved difficult. Most objects are small, far away, and thus difficult to study even with the largest telescopes available. For the small number of objects large enough for detailed compositional study to be feasible, only the surface chemistry, which is clearly not representative of the entire body, is accessible to observation. For detailed understanding of ices and minerals incorporated into bodies in the early outer solar system, short-period comets, which are generally derived from the Kuiper belt, are much better targets for study.

For the first decade of the study of compositions of KBOs, most effort was put into simple cataloging and classifying the surfaces, with little insight into underlying causes. The past decade, however, has seen a maturation of both the data sets available and the physical and chemical insights into their meaning. The chemical composition of KBOs serves a tracer of the physical and chemical processes to which these bodies were subjected at formation and in the time period since. Using studies of surface composition we have gained insight into atmospheric processes, differentiation and cryovolcanism, radiation processing, the effects of giant impacts, and the early dynamical excitation of the Kuiper belt.

In this review we attempt to formulate a coherent picture explaining the compositions of Kuiper belt objects. We show that KBO surface composition divides most strongly by size, with large, medium, and small objects having distinct surface types, and we propose physically and chemically plausible hypotheses for all of these surfaces. We attempt to formulate similar hypotheses for the bulk compositions of KBOs, but we find no satisfactory hypothesis that fits the current data. We end with some thoughts on important open questions that would best allow progress to be made in these areas. 


\section{The largest Kuiper belt objects}

\subsection{Volatiles}

Spectroscopic study of some of the earliest discovered KBOs revealed surfaces that were either spectrally featureless or contained only small amounts of absorption due to water ice. These early spectra of relatively small objects were in marked contrast to the reflectance spectrum of the much larger Pluto, which is rich in absorption features due to $\mathrm{CH}_{4}, \mathrm{~N}_{2}$, and $\mathrm{CO}$ (see Cruikshank et al. 1997; Brown 2002, for reviews), all of which are volatile at the 30-50 K surface temperatures of Pluto. Triton, a body even larger than Pluto and thought to be a captured KBO, has a similar compliment of volatile chemicals on its surface (Brown et al. 1995). The advent of wide field surveys of the outer solar system (Trujillo \& Brown 2003; Brown 2008; Schwamb et al. 2010), however, led to the discovery of objects approaching and even comparable in size to Pluto, many of which are also covered in some of these same volatile chemicals.

Definitive detections of $\mathrm{CH}_{4}$ (a molecule with strong absorption bands in the near-infrared which make the species easy to detect) have now been made on 6 of the 8 largest known KBOs: Triton, Eris, Pluto, Makemake, Sedna, and Quaoar (Brown et al. 1995; Cruikshank et al. 1997; Brown, Trujillo \& Rabinowitz 2005; Licandro et al. 2006; Barucci et al. 2005; Schaller \& Brown 2007a). A 7th - 2007 OR10 - is suspected from indirect evidence to also contain $\mathrm{CH}_{4}$, but no confirming spectrum has yet been obtained (Brown, Burgasser \& Fraser 2011). $\mathrm{N}_{2}$ and CO have weaker absorption bands and are therefore more difficult to detect, so no absorptions due to these species have been detected on any objects other than Pluto or Triton. Figure 1 shows a composite of spectra of some of the volatile-containing KBOs.

The specific volatile species detected on these largest KBOs are not surprising. Of the molecules know to be abundant in comets, $\mathrm{CH}_{4}, \mathrm{~N}_{2}$, and $\mathrm{CO}$ are the only ones that have moderate vapor pressures at the temperatures in the Kuiper belt. Most other major molecules remain in the frozen state on the surface, while some super-volatiles, like noble gasses, have such high vapor pressures that, if they were present, they would be exclusively in the gas phase. $\mathrm{CH}_{4}, \mathrm{~N}_{2}$, and $\mathrm{CO}$, in contrast, can reach an equilibrium between frost on the surface and a molecular atmosphere. This surfaceatmosphere exchange ensures that, when volatile abundances are sufficiently high, the volatile frosts will coat the surface, masking the composition of whatever lies beneath.

The relative abundances on the volatile containing KBOs are not identical. On Makemake, the brightest and most easily studied of the newly discovered large objects, the $\mathrm{CH}_{4}$ absorption features appear significantly different from those on the other volatile-rich KBOs. The absorption lines are broad and saturated, as occurs when the optical path lengths through the solid $\mathrm{CH}_{4}$ are long. The surface producing this spectrum has been modeled as a solid slab of $\mathrm{CH}_{4}$ with voids approximately $\sim 1 \mathrm{~cm}$ apart (Brown et al. 2007a; Eluszkiewicz et al. 2007). On Pluto and Triton, in contrast, small wavelength shifts in the position of the near-infrared absorption features of the $\mathrm{CH}_{4}$ show that the molecule primarily occurs as a minor constituent diluted in an $\mathrm{N}_{2}$ matrix (Cruikshank et al. 1993; 


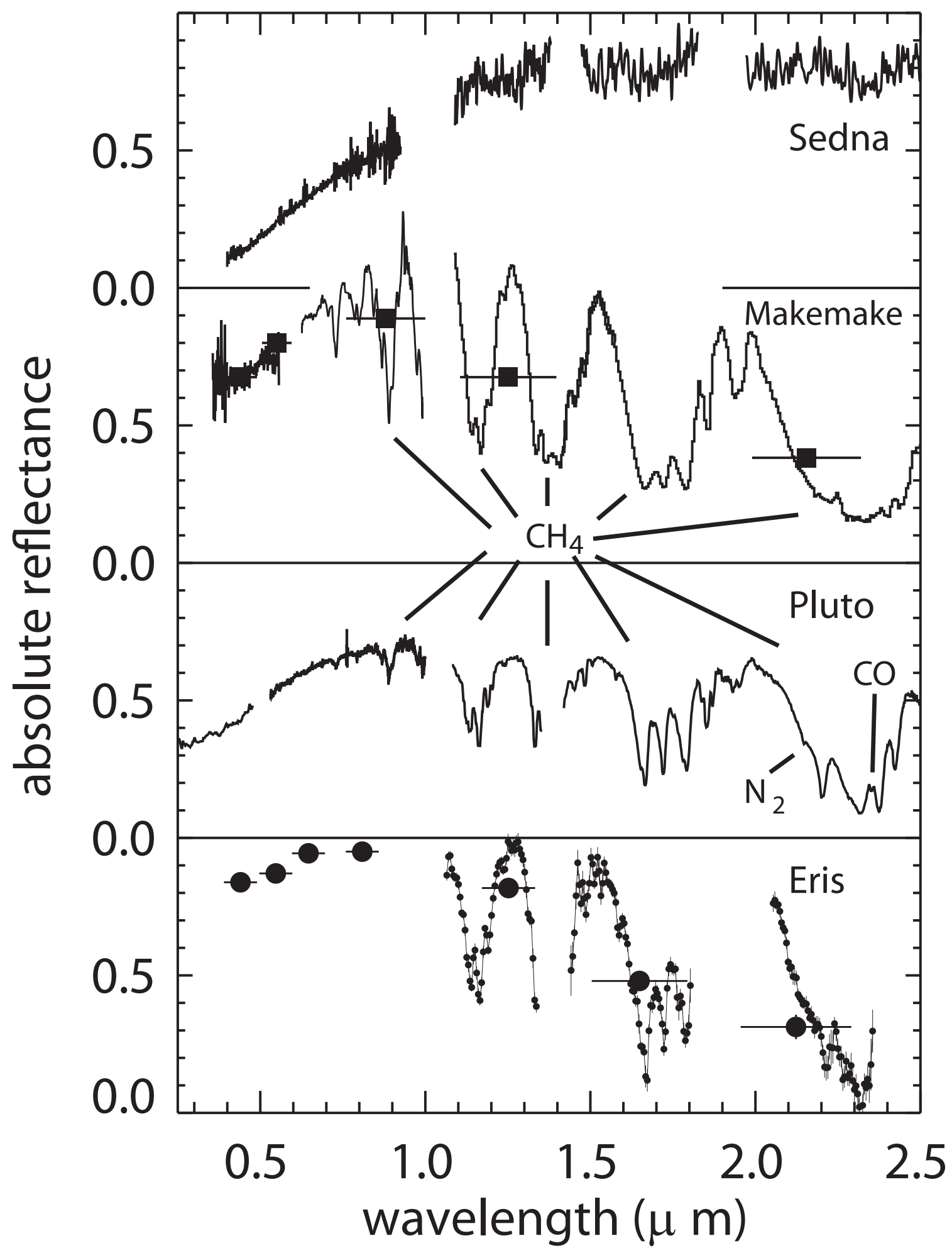

Fig. 1. - Spectra of four of the largest and most volatile rich KBOs. The prominent $\mathrm{CH}_{4}$ absorption lines can be seen on all objects. The weaker $\mathrm{CO}$ and $\mathrm{N}_{2}$ lines can only be detected on Pluto. 
Owen et al. 1993) though regions of pure $\mathrm{CH}_{4}$ frost are also seen to exist (Douté et al. 1999). $\mathrm{CH}_{4}$ is at least an order of magnitude more abundant compared to $\mathrm{N}_{2}$ than it is on Pluto (Brown et al. 2007a). The presence of $\mathrm{N}_{2}$ has nonetheless been indirectly inferred by noting small shifts in the wavelengths of some of the far red $\mathrm{CH}_{4}$ absorption features (Tegler et al. 2008b).

Quaoar, even more dramatically, has such a low surface abundance of $\mathrm{CH}_{4}$ that only the strongest absorption features are detectable, superimposed on a background of water ice absorption (Schaller \& Brown 2007a). The $\mathrm{CH}_{4}$ seems to be a patchy covering on an involatile water ice substrate. The more recently discovered 2007 OR10 is thought to have a similar surface composition to Quaoar, based on its size and its optical colors (see below).

The presence of volatiles on these large objects and even the differences in relative abundances between the volatiles can be explained by a simple model of volatile loss and retention in the Kuiper belt developed by Schaller \& Brown (2007b). In this model all KBOs are assumed to start with typical cometary abundances but then volatile escape to space occurs at a rate determined by the surface temperature and gravity. Volatile loss can occur through a variety of mechanisms on a small body with a vapor-pressure controlled atmosphere, but the slowest mechanism possible is simple Jeans escape from an atmosphere in hydrostatic equilibrium at its surface equilibrium temperature. An updated version of the model results of Schaller \& Brown (2007b) (from Brown, Burgasser \& Fraser (2011)) is shown in Figure 2. In short, volatiles can be retained on objects which are either massive enough to prevent significant Jeans escape or cold enough to prevent significant vapor pressure of the frosts. The three main volatiles behave slightly differently because of differing vapor pressures and molecular masses. $\mathrm{N}_{2}$ or CO have identical molecular masses, so escape identically for identical vapor pressures, but $\mathrm{N}_{2}$ is more volatile, so $\mathrm{N}_{2}$ always has a higher vapor pressure and escapes more quickly. $\mathrm{CH}_{4}$ has the lowest vapor pressure of the three, so generally escapes more slowly, but for high enough temperatures the vapor pressure is sufficiently high that the escape is controlled more by molecular weight, and the lighter $\mathrm{CH}_{4}$ escapes the quickest.

In the model, four important regimes are seen, corresponding nearly precisely to the observations. The largest and coldest objects - Triton, Eris, Pluto, and Sedna - have the ability to maintain their full complement of volatiles. All three volatiles have indeed been detected on Triton and Pluto. On Eris and Sedna direct detection of $\mathrm{N}_{2}$ is difficult owing to the faintness of the objects, the weakness of the single quadrapole $\mathrm{N}_{2}$ absorption line, and the fact that at the low temperatures of the very distant Eris and Sedna, $\mathrm{N}_{2}$ should be in its $\alpha$ state leading to an extremely narrow absorption feature (Tryka, Brown \& Anicich 1995). On Eris, however, subtle shifts in the far red portion of the spectrum again show evidence that some $\mathrm{CH}_{4}$ is diluted within a $\mathrm{N}_{2}$ matrix (Tegler et al. 2010). For Sedna, early observations suggested the presence of an $\mathrm{N}_{2}$ absorption feature, but this feature appeared similar to that on Triton, a broad feature of $\beta$-nitrogen, which would be difficult to explain on the frigid Sedna (Barucci et al. 2005). Other observations have failed to confirm this feature (Barucci et al. 2010). It appears likely that $\mathrm{N}_{2}$, even if abundant on Sedna, would be nearly impossible to detect spectroscopically. CO would be extremely difficult to 


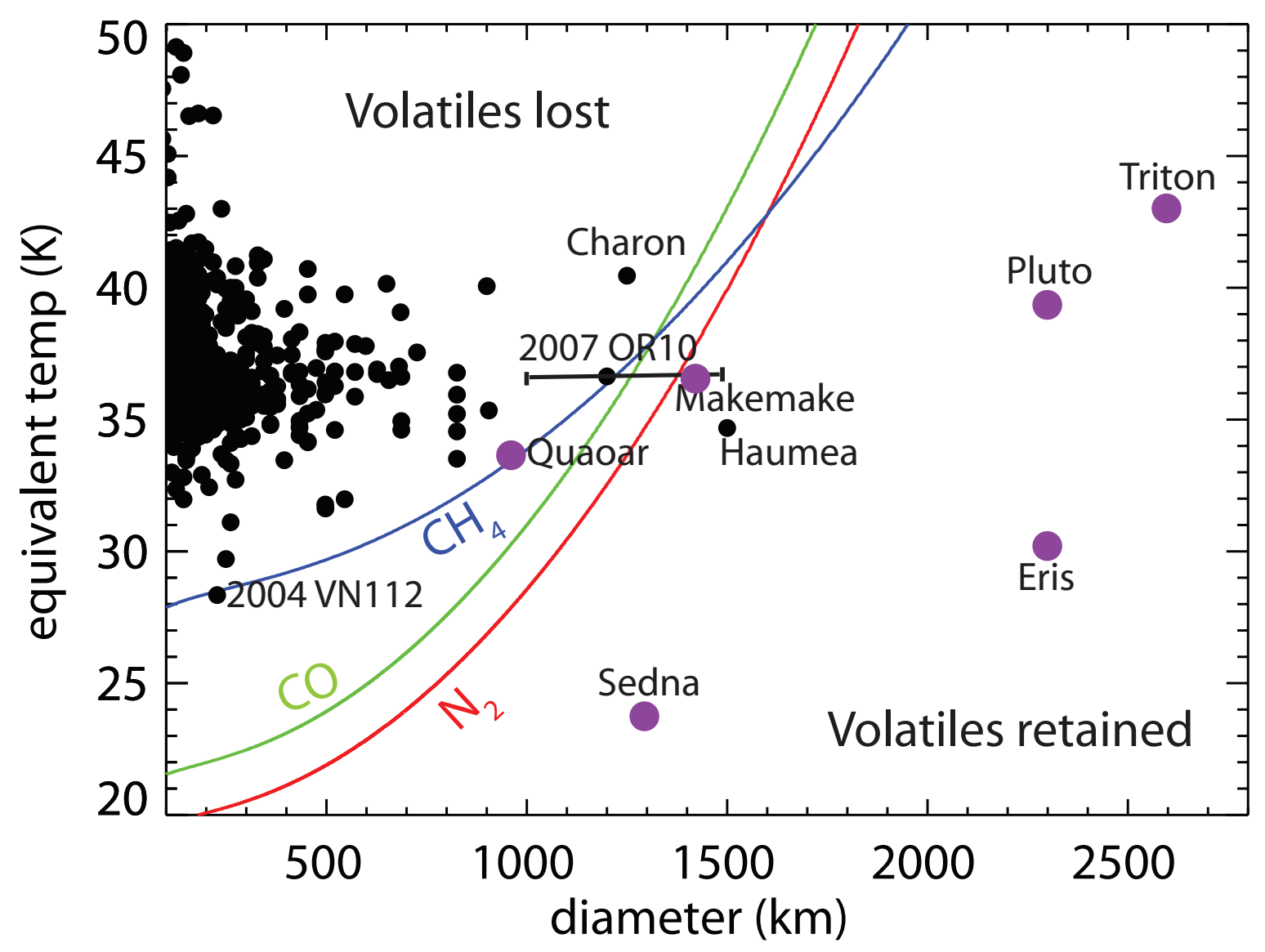

Fig. 2.- Volatile retention and loss in the Kuiper belt. Objects to the left of the $\mathrm{CH}_{4}, \mathrm{CO}$, and $\mathrm{N}_{2}$ lines are too small and too hot to retain any of those surface volatiles over the age of the solar system, while objects to the right can retain those volatiles. All objects shown in purple have had $\mathrm{CH}_{4}$ measured on their surfaces. Some have additionally had $\mathrm{N}_{2}$ or $\mathrm{CO}$ detected. No objects to the left of the lines have had any of these volatiles detected. Methane is suspected, but not confirmed, on 2007 OR10. 
detect on Eris or Sedna, and, to date, no meaningful upper limits have been placed.

The next major regime in the model is occupied only by Makemake. Makemake has just the right size and temperature to be able to retain its $\mathrm{CH}_{4}$, but to be on the edge of not being able to retain $\mathrm{N}_{2}$ and $\mathrm{CO}$. The unusual surface of Makemake appears well explained by this model. With a low abundance of $\mathrm{N}_{2}$ on the surface, $\mathrm{CH}_{4}$ becomes the major constituent and anneals into large slabs, giving rise to the long optical path lengths which saturate the infrared spectrum.

The third regime in the model is the transition where $\mathrm{CH}_{4}$ can only barely be retained and other volatiles are nonexistent. Quaoar and potentially 2007 OR10 are both in this regime. These objects are both sufficiently depleted even in methane that their water ice substrate is visible in regions not covered by methane.

The object 2004 VN112, though likely small, has a perihelion of 47 AU and thus stays cold enough to potentially retain at least $\mathrm{CH}_{4}$. This object is too faint for infrared spectroscopy, so we have no direct indication of its surface composition, and its albedo, and thus, size is unknown. Its optical colors are relatively neutral, however, suggesting that it is not dominated by $\mathrm{CH}_{4}$ irradiation products (see below). Further physical study of this object is clearly warranted.

The final regime within this model is the region in which most of the Kuiper belt resides, where temperatures are too high and masses are too low, so that even with slow Jeans escape, the three main volatiles must be depleted over solar system time scales.

To date, this model has been flawless for determining which objects do and do not retain volatiles in the Kuiper belt (with the important exception of Haumea, which is discussed below). It is interesting - and even unexpected - that such a simple model would work so well. The wellstudied atmospheres of Pluto and Triton, for example, do not conform to the simple assumptions of surface temperature hydrostatic equilibrium and Jeans escape. Perhaps, however, the power of the model is that as volatiles begin to become depleted through faster mechanisms such as hydrodynamic escape, the atmosphere eventually becomes tenuous enough that Jeans escape is the only remaining mechanism functioning. Jeans escape is then so slow compared to other processes that it eventually dominates the total amount of time that it takes for volatile loss.

While Kuiper belt volatiles have mostly been studied from infrared spectroscopy, stellar occultations provide another alternative for study of volatile compositions. Such occultations should be able to provide insights into $\mathrm{N}_{2}$ abundances on Eris, Makemake and Sedna and occultations should provide the best means of looking for volatiles around small distant objects such as 2004 VN112.

\subsection{Radiation processing}

All bare surfaces in the solar system are subject to irradiation by solar wind, UV photons, and cosmic rays, all of which are capable of inducing chemical changes in the surfaces. The surface colors of most KBOs are likely set by this irradiation (see below), but, for many years, the only radiation 
products thought to be seen in the outer solar system were tholins - the reddish involatile residues of long term irradiation of hydrocarbon and nitrogen containing compounds. Many other simpler molecular radiation products of $\mathrm{N}_{2}, \mathrm{CO}$, and $\mathrm{CH}_{4}$ were synthesized in laboratories and predicted to be present on Pluto or Triton (Hudson et al. 2008), but the low abundances, difficult-to-observe spectral features, and lack of adequate data prevented any positive detections.

The discovery of the large slabs of $\mathrm{CH}_{4}$ on Makemake, however, also led to the first direct detection of simple radiation products. The saturation of the strong $\mathrm{CH}_{4}$ absorption line around $2.3 \mu \mathrm{m}$ allows deviations from the $\mathrm{CH}_{4}$ spectrum to be easily identified. The strongest deviations are clearly due to the presence of $\mathrm{C}_{2} \mathrm{H}_{6}$ (Fig 3). $\mathrm{C}_{2} \mathrm{H}_{6}$ forms from the combination of two $\mathrm{CH}_{4}$ molecules which have each had a hydrogen atom removed by irradiation, and it is the first stable molecule to form in the presence of $\mathrm{CH}_{4}$ irradiation (Bennett et al. 2006).

Makemake, which is dominated by $\mathrm{CH}_{4}$ rather than $\mathrm{N}_{2}$, as on Pluto, is an ideal laboratory for understanding this radiation processing. On Pluto, creation of $\mathrm{C}_{2} \mathrm{H}_{6}$ is stymied by the dilution of the $\mathrm{CH}_{4}$ in a $\mathrm{N}_{2}$ matrix. Radiation can remove a hydrogen atom, but another $\mathrm{CH}_{3}$ radical will rarely be present to form $\mathrm{C}_{2} \mathrm{H}_{6}$. Nonetheless, recent observations have suggested a small amount of $\mathrm{C}_{2} \mathrm{H}_{6}$ on Pluto, as well (DeMeo et al. 2010; Merlin et al. 2010a), presumably coming from the small regions of pure $\mathrm{CH}_{4}$ which have been detected (see above).

Quaoar, though having significantly less coverage of $\mathrm{CH}_{4}$ on its surface than Makemake, also appears to have detectable $\mathrm{C}_{2} \mathrm{H}_{6}$ embedded within its surface, which would be expected for an object with $\mathrm{CH}_{4}$ and little to no $\mathrm{N}_{2}$. Quaoar is also unusual for having both deep water ice absorption features and a very red color. The radiation processing of $\mathrm{CH}_{4}$ appears likely to be the cause of this coloration; long-term irradiation of $\mathrm{CH}_{4}$ will cause the ice to turn red (Brunetto et al. 2006). 2007 OR10, which appears in a similar regime of volatile retention as Quaoar, has a similar very red surface combined with deep water ice absorptions, leading to the hypothesis that it, too, contains $\mathrm{CH}_{4}$, and, presumably, its radiation products.

After the creation of $\mathrm{C}_{2} \mathrm{H}_{6}$, continued irradiation leads to $\mathrm{C}_{2} \mathrm{H}_{2}$ (acetylene) and $\mathrm{C}_{2} \mathrm{H}_{4}$ (ethylene). These molecules have increasingly difficult to detect spectral features and have not been detected on Makemake - or any other object in the solar system - to date, but it is likely that they are present as temporary products which eventually lead to the red coloration of the long-chain hydrocarbons.

Orcus is similar in size to Quaoar but warmer, and thus is not expected to retain volatiles. Delsanti et al. (2010) nonetheless suggest the detection of $\mathrm{C}_{2} \mathrm{H}_{6}$, an involatile irradiation product on this surface. While it is possible to imagine a scenario in which $\mathrm{CH}_{4}$ irradiation leads to irradiation products which then stay behind as a lag deposit after the $\mathrm{CH}_{4}$ has been lost, it is difficult to construct a scenario in which the surface does not also retain the red coloration of more complex irradiation products. Orcus is instead amongst the most neutrally colored KBOs. We suspect the higher signal-to-noise spectra of Orcus will confirm a lack of methane irradiation products on its surface. 


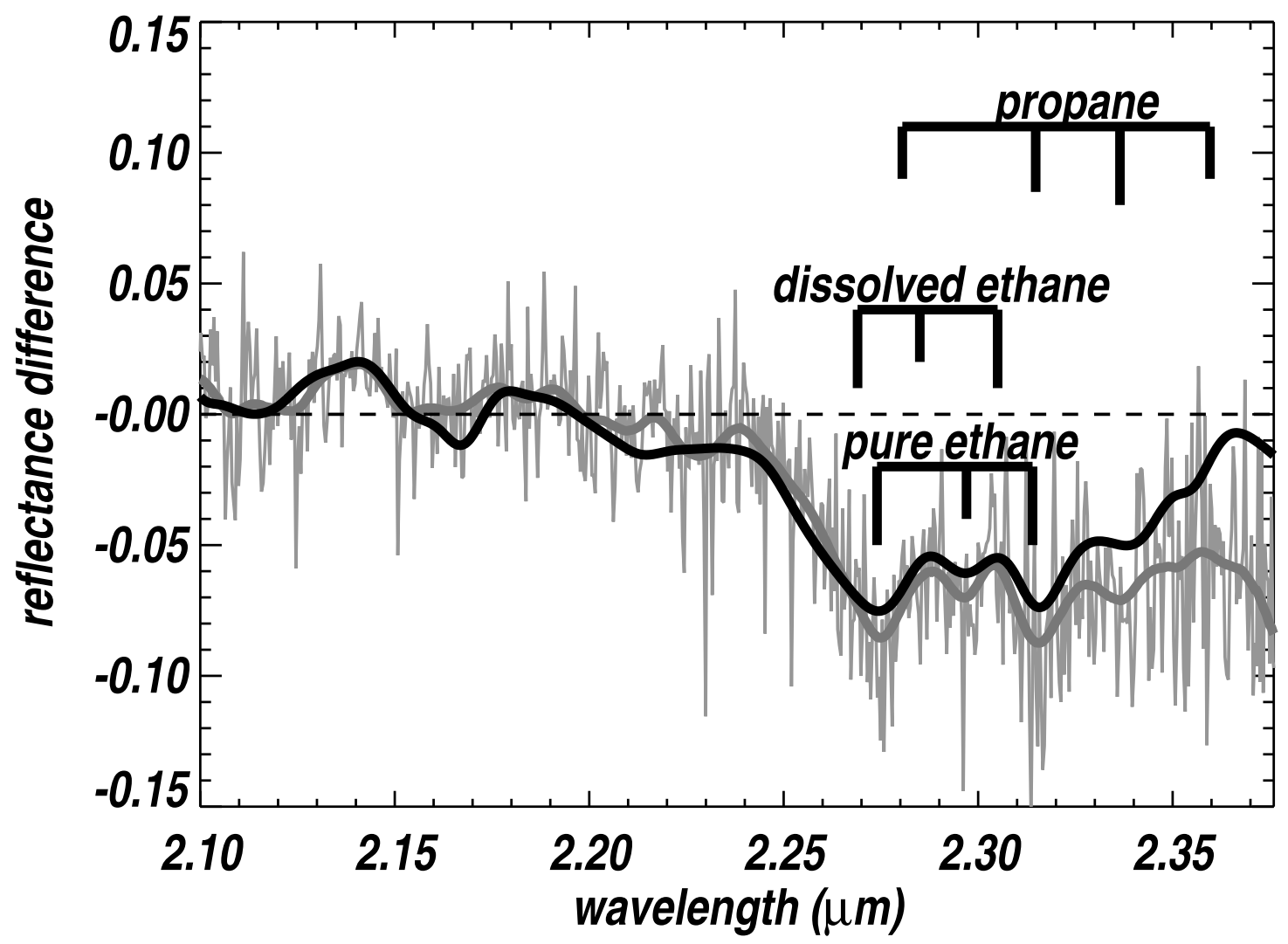

Fig. 3.- Detection of pure ethane on Makemake. The data show the difference between the data and a $\mathrm{CH}_{4}$ model of the surface. The deviations from the $\mathrm{CH}_{4}$ model show the clear signature of pure ethane ice, an expected irradiation product of $\mathrm{CH}_{4}$. Evidence for additional absorption is present, but no positive identification of the species has yet occurred. 


\subsection{Haumea and its family}

Haumea is unique in the solar system. It is the fastest rotating and most elongated gravitationally bound object in the solar system, has a density nearly that of rock (Rabinowitz et al. 2006), and is surrounded by two moons (Brown et al. 2005, 2006). Though it is one of the largest objects in the Kuiper belt and would be predicted by the simple model above to contain abundant volatiles, its surface is instead dominated by what appears to be nearly pure water ice (Truillo et al. 2007). The moons likewise appear to have surfaces of pure water ice (Barkume. Brown \& Schaller 2006; Fraser \& Brown 2009), and a dynamical family of KBOs with pure water ice surfaces exists in orbits separated from Haumea by only a few hundred meters per second (Fig 4).

While Haumea is large enough to be differentiated, so that we can imagine an icy outer layer surrounding a predominantly rocky body, the known dynamical family members and the satellites range in size from $\sim 70$ to $\sim 370 \mathrm{~km}$ (assuming 0.7 albedos), likely too small to be differentiated. For these objects, the fact that the surfaces appear to be nearly pure water ice strongly suggests that these objects are nearly pure water ice in their interiors, also. In particular, the mutual orbital perturbations of the two Haumea satellites show that their masses are consistent with them having bulk densities even lower than $1 \mathrm{~g} \mathrm{~cm}^{-3}$ (Ragozzine \& Brown 2009).

All of these characteristics are likely the product of a single oblique giant impact onto a differentiated proto-Haumea earlier in the history of the solar system (Brown et al. 2007b). Prior to impact, the proto-Haumea would have been differentiated into a rocky core, a relatively pure water ice mantle, and a crust of volatiles that had been driven to the surface and their irradiation products. The oblique impact then gave Haumea its fast rotation, causing its extreme elongation. In addition, the impact must have blasted off a significant fraction of the proto-Haumea's icy mantle, which then became the satellites and the collisional family.

In this scenario, the nearly pure water ice surface of Haumea (along with its high density) occurs because we are viewing the exposed layers of the interior pure icy mantle. The nearly pure water ice surfaces of the dynamical family members and the satellites occur because they are fragments of the interior of the mantle. Long term irradiation of these pure ice fragments does not cause any of the reddening or darkening that would be expected if any hydrocarbons were present. Fragments of the crust, which should have contained more irradiated hydrocarbons, presumably were created also, but the volume of crust is much smaller than that of mantle, and there is currently no method

for positive identification of non-ice components of Haumea, so to date only mantle fragments are known.

Even if the post-impact Haumea and its collisional family originally began with nearly pure water ice surfaces, the fact that these surfaces remain relatively uncontaminated with dust, fragments of dark impactors or irradiated hydrocarbons is a surprise. It has even been suggested that the collision must have been relatively recent for the surfaces to appear so pure (Rabinowitz et al. 2008). Dynamically modeling of the diffusion of Haumea with the 12:7 mean motion resonance with Neptune suggests, however, that the collision occurred billions of years ago (Ragozzine \& Brown 


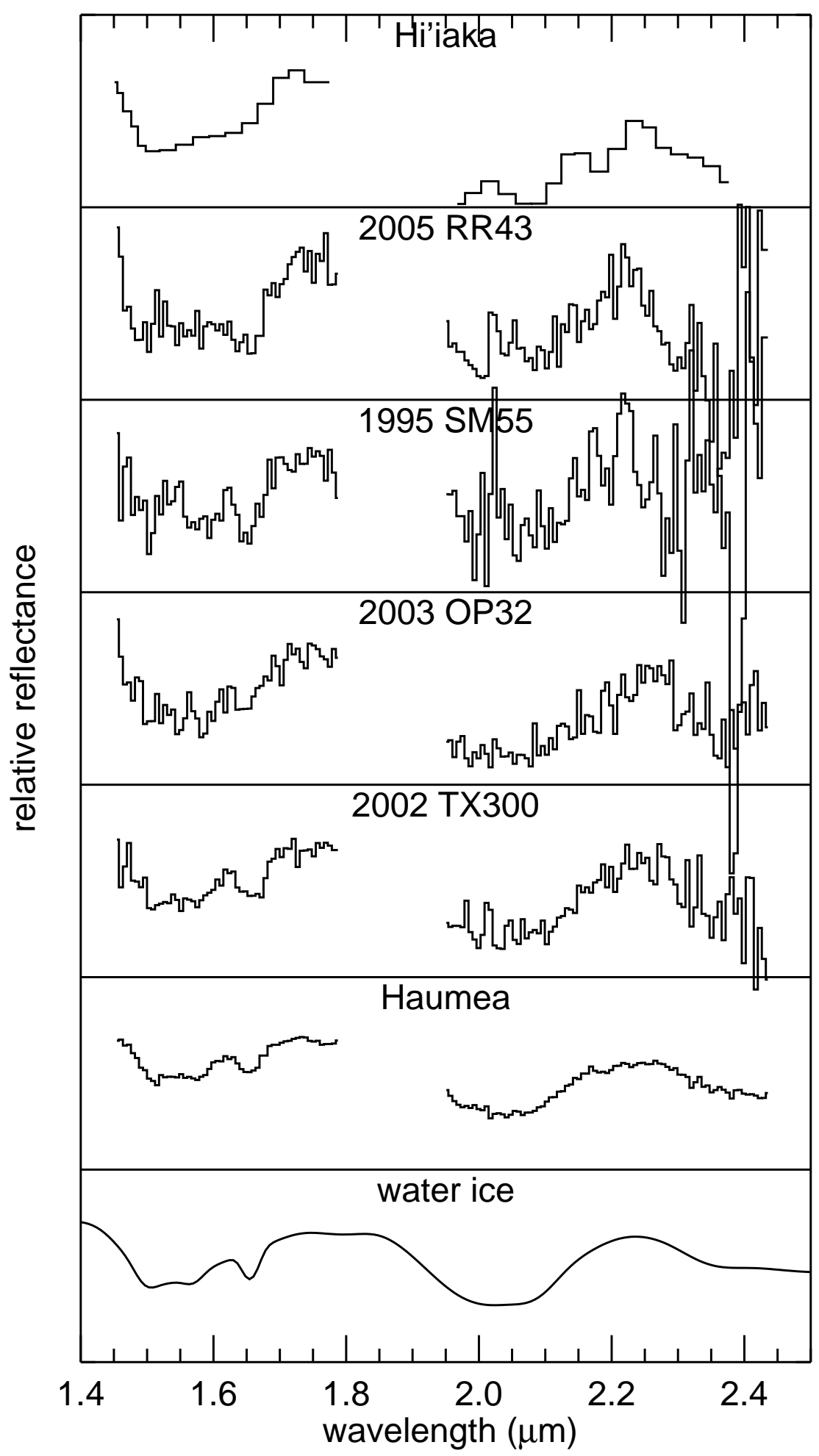

Fig. 4.- Near infrared reflectance spectra of Haumea, its satellite Hi'iaka, and some members of its collisional family, compared to a model of a laboratory spectrum of pure ice. No objects with comparably deep water ice absorption features are found anywhere in the Kuiper belt other than the dynamical vicinity of Haumea. The spectra are consistent with nearly pure water ice. In all cases with sufficient signal-to-noise, the spectrum shows the $1.65 \mu \mathrm{m}$ absorption feature of 
2007). Modeling of collision probabilities suggests that the collision must have occurred near the beginning of the solar system (Levison et al. 2008). Pure water ice surfaces in the Kuiper must be capable of staying relative pristine for billions of years. While the reasons for this longevity are not clear, we will use this empirically determined fact below when discussing water ice on the mid-sized objects.

In addition to being relatively pristine, the spectrum of water ice on Haumea and its family members always contains the $1.65 \mu \mathrm{m}$ absorption feature due to crystalline water ice. While this $1.65 \mu \mathrm{m}$ feature appear ubiquitous throughout the outer solar system, its presence is perhaps surprising. Irradiation studies suggest that crystalline water ice should turn to amorphous water ice on relatively short time scales unless some heating is applied to recrystallize the water ice. This $1.65 \mu \mathrm{m}$ feature is thus often taken as evidence of some type of internal heating, from cryovolcanism (Jewitt \& Luu 2004) to radioactivity and tidal forces (Dumas et al. 2011). The fact that the 1.65 $\mu \mathrm{m}$ absorption can be seen even on Haumea family members as small as 1995 SM55, with an estimated size of $\sim 180 \mathrm{~km}$ in diameter (assuming an identical albedo to Haumea) demonstrates, however, that no such internal mechanism is required for the appearance of this feature. These objects are far to small to maintain the liquid water beneath their surface that would be necessary to support any current cryovolcanism and as fragments of the icy mantle of Haumea they are thoroughly lacking in the rocks that would give rise to radioactive heating. It seems clear, even if not understood, that the appearance of the $1.65 \mu \mathrm{m}$ absorption feature of crystalline water ice does not contain any information about internal processes, but rather contains new information about the physics of crystallization under these conditions.

\section{Mid-sized objects}

While the surface compositions of the largest object appear well understood and even predictable, less attention has been paid to the mid-size $\sim 500$ - $1000 \mathrm{~km}$ diameter KBOs which are just a little too small to be able to have retained surface volatiles. In some ways, these objects form a more interesting class than the larger objects, as these objects do not have a frosty veneer hiding the inherent surface composition. Unfortunately, however, while high quality spectroscopy of the largest and brightest objects can be readily obtained, these mid-sized objects are considerably more difficult to observe even with the largest telescopes in the world. Major observing campaigns with the Keck telescope (Barkume. Brown \& Schaller 2008; Brown, Schaller \& Fraser 2011b) and the VLT (Guilbert et al. 2009; Barucci et al. 2011) have provided much of what we know of the surface compositions of these objects. 


\subsection{Water ice}

Barucci et al. (2011) and Brown. Schaller \& Fraser (2011b) both analyze extensive collections of high quality spectra of KBOs and their progeny. In Barucci et al. (2011) the possibility of water ice in VLT spectra is first assessed by simply calculating the depth of a potential $2 \mu \mathrm{m}$ absorption feature and then followed by detailed modeling including many different components. Brown. Schaller \& Fraser (2011b) concentrate on a examining the possibility of water ice in Keck spectra by fitting a simple water ice plus sloped continuum model to the near infrared spectrum. The VLT and Keck results are in agreement both on the broad results and on most of the individual objects.

In Figure 5, we show the water ice spectral fraction - a measure of the depth of the water ice absorption in the spectrum - as a function of the absolute magnitude (a proxy for diameter) of KBOs and also of centaurs (see below), from the Brown. Schaller \& Fraser (2011b) sample. A clear trend is evident: brighter than an absolute magnitude of $H=3$, all KBOs have deep water ice absorption (water ice spectral fraction $>0.2$ ). Fainter than an absolute magnitude of $H=5$, deep water ice absorption is never seen.

While such a correlation of absolute magnitude and presence of water ice might be expected simply from the increased albedo of objects with more water ice on their surfaces, Spitzer radiometry has shown that the $H>3$ KBOs are indeed smaller than the $H<3$ KBOs (Stansberry et al. 2008). Somewhere between the $\sim 650 \mathrm{~km}$ diameter of Ixion and the $\sim 900 \mathrm{~km}$ diameters of Quaoar and Orcus, KBOs appear to dramatically change in surface composition.

The change in surface composition is clearly not monotonic with size. Ixion, 2002 UX25, 2002 AW197, 2004 GV9, and 2003 AZ84 have $650 \mathrm{~km}$ diameters within their uncertainties (Stansberry et al. 2008), but only 2003 AZ84 has a large abundance of water ice on the surface. Likewise, the smaller $2005 \mathrm{RM} 43$, which would have a diameter of $\sim 520 \mathrm{~km}$ if it has the same albedo as 2003 AZ84, has water ice absorption as deep as the larger objects Quaoar and 2007 OR10, while the similarly-sized objects Varuna and Huya have much smaller absorption depths.

Smaller than the $\sim 520 \mathrm{~km}$ size of 2005 RM43, however, no (non-Haumea family member) KBO has been found with strong water ice absorption. Few objects in this range are bright enough for high quality spectroscopy (1996 TP66, with a diameter of $\sim 180 \mathrm{~km}$ for an assumed 0.1 albedo is the smallest KBO with a well measured spectrum), however. Nonetheless, spectroscopic evidence from centaurs and photometric evidence from smaller KBOs (see below) suggests that this trend continues, and that between 500 and $700 \mathrm{~km}$ in diameter KBOs transition from typical surfaces of small KBOs to those dominated by absorption due to water ice.

Additional evidence for a surface change in this diameter range comes from measurements of albedos. Though the size and albedo data available from Spitzer photometry have large uncertainties, a jump in albedo at this size range is apparent. The same effect is see in the cumulative absolute magnitude distribution of the large objects. While this distribution follows a power law 


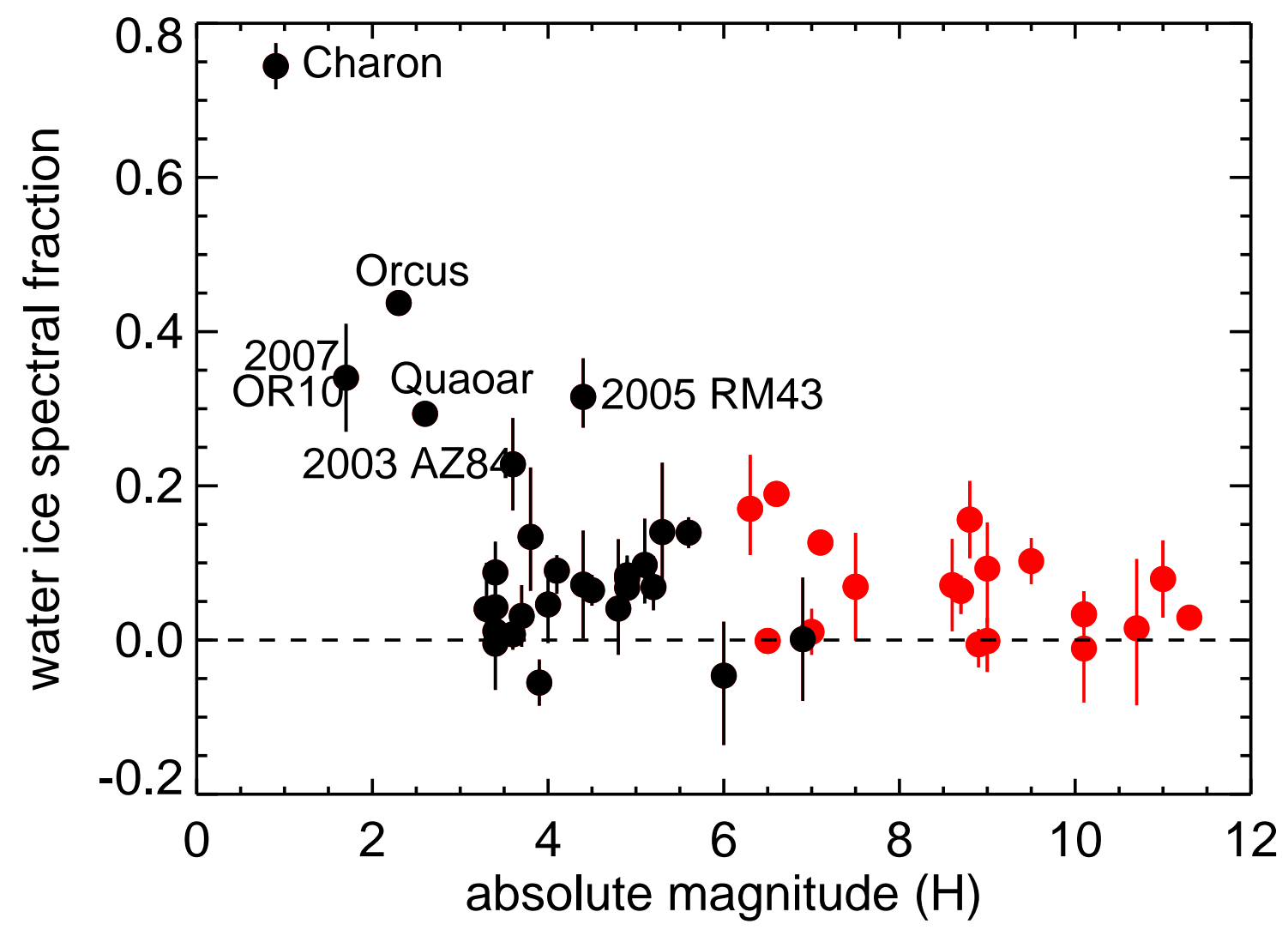

Fig. 5. - The water ice spectra fraction - a measure of the amount of water ice absorption in an object spectrum - as a function of absolute magnitude. Absolute magnitude is a measure of the intrinsic brightness of the object, which is related to size. The largest objects - on the left of the plot - have the smallest absolute magnitudes. A clear trend is seen for the largest objects to have the deepest water ice absorption spectra. The transition around $H \sim 4$ from moderate to deep water ice absorption is a potential indicator of the size at which interior oceans caused surface ice flows in the past. The water ice fraction on smaller objects is indistinguishable from that on the even smaller centaurs. 
from absolute magnitudes of 3 until at least 4.5, for objects brighter than $H=3$, the cumulative number distribution is larger than would be expected from this power law, a clear sign of the increased albedos of these mid-sized objects (Brown 2008). Implications for this surface change are discussed below.

\subsection{Ammonia}

Of these mid-sized objects with strong water ice absorption features, two have evidence for a $2.25 \mu \mathrm{m}$ absorption feature due to ammonia. Ammonia was first detected on Charon (Brown \& Calvin 2000; Cook et al. 2007; Merlin et al. 2010a) and later suggested to be present also on Orcus (Barucci et al. 2008b). In both cases, the presence of ammonia was postulated to be due to the flow of ammonia-rich interior liquid water on to the surface of the object at some point in the past. Detailed models of the interior structure and evolution of these two bodies have suggested that such a scenario appears reasonable (Cook et al. 2007; Delsanti et al. 2010).

Ammonia has not been detected on the other water ice-rich objects, but for Quaoar a bright object where high quality spectra can be obtained, the $2.3 \mu \mathrm{m}$ region of the spectrum where ammonia has its only detectable near-infrared feature is instead dominated by the strongest absorption feature due to $\mathrm{CH}_{4}$. Ammonia has additional absorption features beyond $3 \mu \mathrm{m}$ which could be distinguished from $\mathrm{CH}_{4}$ but spectral measurement at these wavelengths awaits larger ground or space-based telescopes. The smaller water-ice-rich objects 2003 AZ84 and 2005 RM43 have spectra with insufficient signal-to-noise to detect ammonia. Larger telescopes will again be required.

Based on the likely presence of ammonia on Charon and Orcus, and the sharp increase in water ice absorption with larger size for these objects, we hypothesize that on these largest objects the presence of water ice - whether ammonia is detectable or not - is a remnant of past liquid flows on the surface. We predict that for these objects ammonia will always be detected when sufficient signal-to-noise is available. The liquid flows need not be recent - the purity of the water ice on the surface of Haumea and its family shows that water ice can remain pristine through the age of the solar system - but the liquid flows must have occurred after the volatiles whose irradiation would cause coloration (see below) have all escaped. The increase in water ice absorption with size would be a natural consequence of the larger interior liquid reservoirs of larger objects. The sharp increase in water ice absorption starting at diameters around $\sim 650 \mathrm{~km}$ gives an important clue into the physics of liquid interiors and surface water flows. 


\section{Small objects: spectroscopic constraints}

\subsection{Kuiper belt objects}

Many objects smaller than the size where strong water ice absorption is present are statistically consistent with having no water ice on their surface, but there is a significant prevalence in the data for positive detection of water ice. Random and known systematic errors would not produce a bias towards water ice detection. Indeed, we regard the nearly complete lack of objects with negative water ice fraction as an indication of the robustness of our method. We conclude, therefore, that even the low level of water ice fraction detected in the majority of the objects is a real indication that water ice at a low level is common even on the objects smaller than Ixion.

2002 VE95, the smallest Kuiper belt object with a very high quality spectrum $(\sim 330 \mathrm{~km}$ diameter assuming a 0.1 albedo), shows clear evidence of crystalline water ice on its surface

(Barkume, Brown \& Schaller 2008; Barucci et al. 2006). Indeed, the $1.65 \mu \mathrm{m}$ absorption feature of crystalline water ice can always be detected when the signal-to-noise is sufficiently high.

Based on their smaller sizes, it seems likely that the water ice on these smaller objects is not caused by liquid flows on the exterior, but rather by the exposure of crustal water ice. An important test of this expectation would be that these smaller KBOs should not show the presence of ammonia on their surfaces. Currently feasible spectroscopy cannot achieve the signal-to-noise required to make this test, however.

\subsection{Centaurs}

KBOs smaller than those discussed above are too faint for high quality spectroscopy even with the largest telescopes in the world. To understand the compositions of these objects, we have to resort to observational proxies. One important proxy has been spectroscopic observations of Centaurs. Centaurs are former KBOs which have been perturbed onto short-lived planet-crossing orbits. Being much closer to the sun than typical KBOs, they are brighter and easier to study in detail.

Spectroscopically, the Centaurs appear indistinguishable from the smallest KBOs whose spectra can be measured: objects contain either no detectable absorption features, a small amount of absorption due to water ice, or (in one case) absorption due to water ice and methanol. Figure 5 includes water ice absorption depth for a large sample of Centaurs, compared to KBOs. No discernible difference can be seen between the largest of the centaurs and the smallest of the measured KBOs. The amount of water ice absorption seen on the surface of a centaur does not appear to correlate with anything, including perihelion, semimajor axis, optical color, dynamical lifetime, or activity. Interestingly, while much speculation has occurred about Centaur surface evolution as objects move closer to the sun and begin heating, infrared spectroscopy shows no such evidence of any change in the distribution of water ice absorption depth. 


\subsection{Methanol}

An absorption band at $\sim 2.27 \mu \mathrm{m}$ was first detected on the bright centaur Pholus (Davies. Svkes \& Cruikshank 1993), and Cruikshank et al. (1998) present the case that this band is plausibly due to the presence of methanol, though they point out that the identification is not unique and that other low molecular weight hydrocarbons or photolytic products of methanol might fit the spectrum equally well. Pholus is one of the reddest objects in the solar system, again fitting our picture of optical colors of irradiated hydrocarbons well.

Based on lower signal-to-noise spectra with properties which resemble the water-ice-plusmethanol spectrum of Pholus, the presence of methanol was suggested on the KBOs 1996 GQ21 and 2002 VE95 (Barucci et al. 2008a). To examine this possibility more closely, we combine the Keck spectra of these two objects to increase signal-to-noise and consider the presence of methanol. While the signal-to-noise remains low, the presence of absorption features similar to those on Pholus is certainly plausible. Both objects, like Pholus, are red.

A handful of other KBOs have recently been reported to also have absorption features near $2.27 \mu \mathrm{m}$, but, unlike Pholus, 1996 GQ21 and 2002 VE95, to not have absorption due to water ice (Barucci et al. 2011). The signal-to-noise in the spectral region are low, so it is difficult to determine if the absorption features are real. To examine the possibility that a $\sim 2.27 \mu \mathrm{m}$ absorption feature occurs on faint KBOs, we first examine all of the KBOs and centaurs in the Keck sample (which includes none of the potential methanol objects from the VLT sample). We find that a small number of objects have absorption features at or near the $2.27 \mu \mathrm{m}$ methanol absorption line, but no single spectrum is sufficiently reliable in this region to assert a positive detection. To increase the signal-to-noise, we sum the spectra of all of the KBOs and centaurs in the Keck sample except for those with water ice absorption at the level of that seen on 2003 AZ84 or deeper and those already suspected to contain methanol-like features (1996 GQ21 and 2002 VE95). This combined spectrum shows residual absorptions due to water, as would be inferred from the positive detections on most objects. The only major deviation from the water ice spectrum occurs at precisely the wavelength of the feature seen on Pholus and suspected on 1996 GQ21 and 2002 VE95. We conclude that the methanol-like feature is indeed present at a low-level on KBOs even though the feature cannot be reliably identified in individual spectra (Figure 6).

No hypothesis has ever been formulated for the sporadic presence of methanol on KBOs or centaurs, other than to point out that methanol is common in cometary comae, so it is expected to be present in the interior of KBOs. Its presence is less expected on the surface of KBOs, however, as the absorption features of hydrocarbon ices quickly degrade under irradiation while the remnants

turn red (Brunetto et al. 2006). Visible methanol absorption features suggest that the methanol has only recently been exposed at the surface, perhaps as a result of a collision exposing the subsurface. One prediction of this suggestion would be that the amount of methanol observed would vary as different faces of the object were observed. While such a test is possible in principle, in practice spectroscopy of these faint objects is sufficiently difficult that variation would be difficult to prove. 


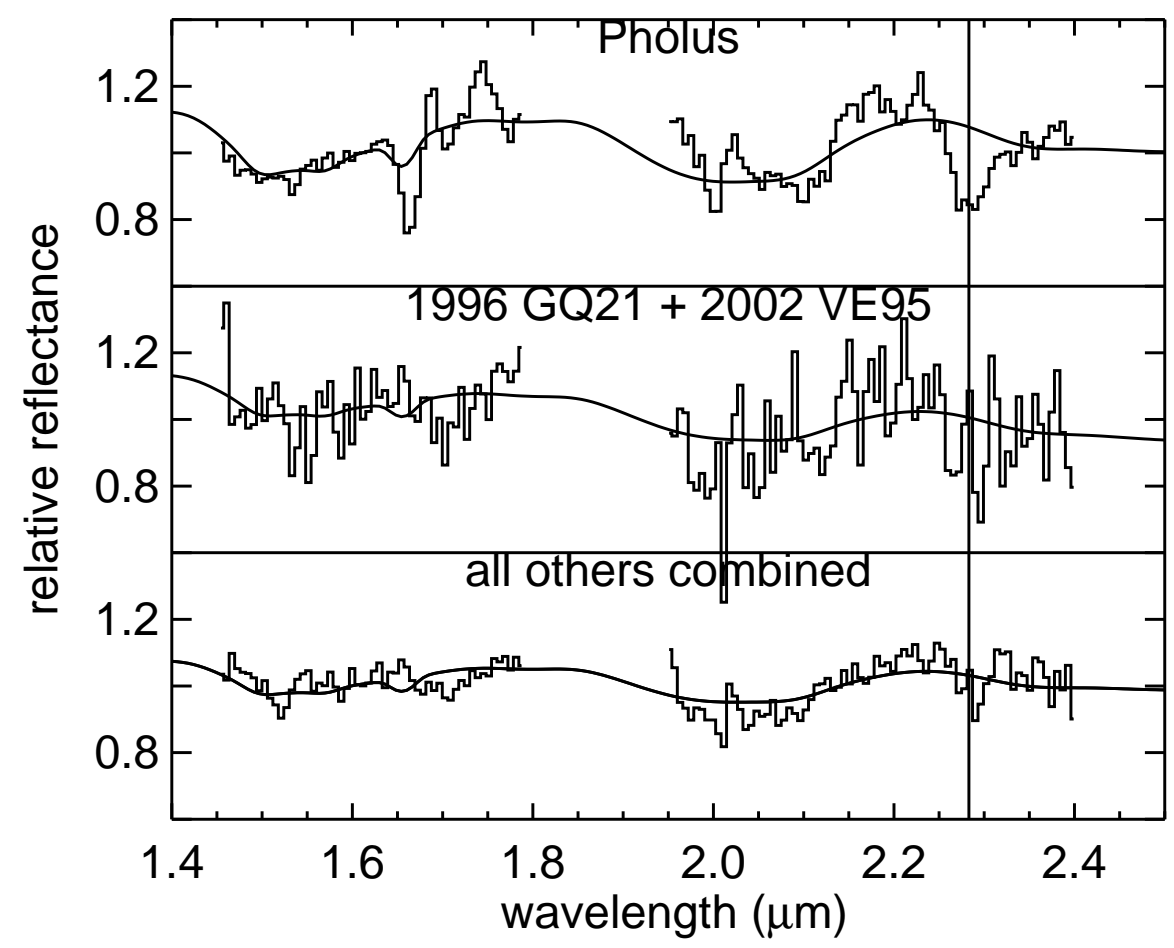

Fig. 6. - The region of the near-infrared spectrum containing the $2.27 \mu \mathrm{m}$ absorption feature attributed to methanol. The feature can clearly be seen on Pholus, superimposed on a small amount of water ice absorption. The combined (to increase signal-to-noise) spectrum of 1996 GQ21 and 2002 VE95 shows hints of a feature at the same location. The sum of $38 \mathrm{KBO}$ and centaur spectra, none of which individually show clear evidence for the feature, clearly shows a feature at the same 
If, however, irradiation preferentially turned the regions with exposed methanol red, these objects should perhaps show color variation with rotation, something which is otherwise rarely observed in objects of this size (Sheppard, Lacerda \& Ortiz 2008).

\subsection{Silicates}

In addition to ices and their irradiation products, we should expect that KBOs that are small enough to be undifferentiated should expose some of their rock component on the surface. While silicates such as olivine or pyroxene are often included in detailed models of KBO spectra (i.e. Merlin et al. 2010b; Barucci et al. 2011) no specific absorptions are easily observable: olivine, for example, has a broad absorption centered at around $1 \mu \mathrm{m}$, where observations are usually poor. These silicates are thus included in models to fit the overall spectral shapes over the $\sim 0.6-1.2 \mu \mathrm{m}$ range.

Mid-infrared observations have the possibility of positively identifying silicate emission in the 10 and $20 \mu \mathrm{m}$ region on KBOs. To date only the centaur Asbolus has had a spectrum measured with sufficient signal-to-noise to even detect these spectral regions, and, in this case, emissivity peaks around 10 and $20 \mu \mathrm{m}$ are indeed seen, similar to that observed in Trojan asteroids (Emery, Cruikshank \& van Cleve 2006; Barucci et al. 2008a), and interpreted to be due to finegrained silicates. Future observations will require improved space-based mid-infrared facilities, but characterization of actual silicate composition is indeed possible.

While olivines or pyroxenes cannot be specifically identified in the visible to near-infrared range, there have been a few reports of shallow broad absorption features in the visible wavelength range similar to absorptions seen on some asteroids. On asteroids these are generally attributed to aqueously altered silicates. Confirmation of these features has been difficult; the absorptions are subtle and have frequently appeared changed or absent upon reobservation of the same object (Fornasier et al. 2004; de Bergh et al. 2004; Fornasier et al. 2009; Alvarez-Candal et al. 2008; Lazzarin et al. 2003) While these changes are usually attributed to rotational variability, it is worth noting that this speculation has never been verified. Indeed, for one object observations over half of a rotational period showed no signs of the visible absorption features.

While it is possible that these difficult-to-confirm features are a product of sporadic systematic error, the possibility of the existence of aqueously altered silicates is an interesting one to consider. The presence of liquid water on surfaces in the Kuiper belt might seem surprising, but hydrous materials seem to be present in small comets, interplanetary dust particles, and debris disks (de Bergh et al. 2004). If these detections are indeed real, the most surprising thing about them, perhaps, is that they are uncommon. 


\section{Small objects: photometric constraints}

Small objects in the Kuiper belt are too faint to detect spectroscopically, but photometric measurements can still give information - albeit limited - about the surfaces of these objects. To date, the single most robust conclusion based on photometry is that Kuiper belt surfaces are diverse. Large surveys of KBO optical colors have found a wider range of surface colors in the Kuiper belt than in any other small body population in the solar system. The colors are uncorrelated with most dynamical or physical properties (see review in Doressoundiram et al. 2008). A few systematic patterns have been found, however, which are important clues to understanding the surface compositions of these outer solar system objects.

\subsection{The bifurcated colors of centaurs}

The range of centaur optical colors generally covers the same wide range of colors found in the Kuiper belt, but the centaurs are deficient in colors in the middle part of the range, giving the distribution of centaur optical colors a bimodal appearance (Tegler et al. 2008a) with a neutral and a red clump of objects. Interestingly, this bifurcation is not seen only in the centaurs, but it appears to extend to all objects with low perihelion distance whether the objects are dynamically unstable or not. This result immediately suggests that the bifurcation in the optical colors is somehow formed through the increased heating or irradiation experienced by lower perihelion objects.

The H/WTSOSS (Hubble/WFC3 Test of Surfaces in the Outer Solar System) survey which used the Hubble Space Telescope to extend optical photometry of centaurs (and other low perihelion

objects) into the near-infrared (Fraser \& Brown 2011) found that the neutral and red clumps do not consist of two groups with identical surfaces, but rather are best described by two groups that fall along two separate mixing lines. The neutral clump of objects consists of a mixture of a nearly neutrally reflecting material and a slightly red material, while the red clump of objects consists of a mixture of the same neutrally reflecting material and a much red material (Fig 7).

While three color photometry is generally incapable of identifying specific ices or minerals, Fraser \& Brown (2011) find that the neutral component common to all centaurs is consistent with some of the same hydrated silicates suggested from the optical spectroscopy discussed above. Significantly more spectral work is required, however, to further explore this possibility.

\subsection{The uniform colors of the cold classical KBOS.}

While most of the rest of the Kuiper belt appears to be composed of essentially the same distribution of neutral to red objects (Morbidelli \& Brown 2005; Doressoundiram et al. 2008) one dynamical region stands out for its homogeneous composition. The cold classical Kuiper belt was first identified as a dynamically unique region of the Kuiper belt - a difficult-to-explain overabun- 


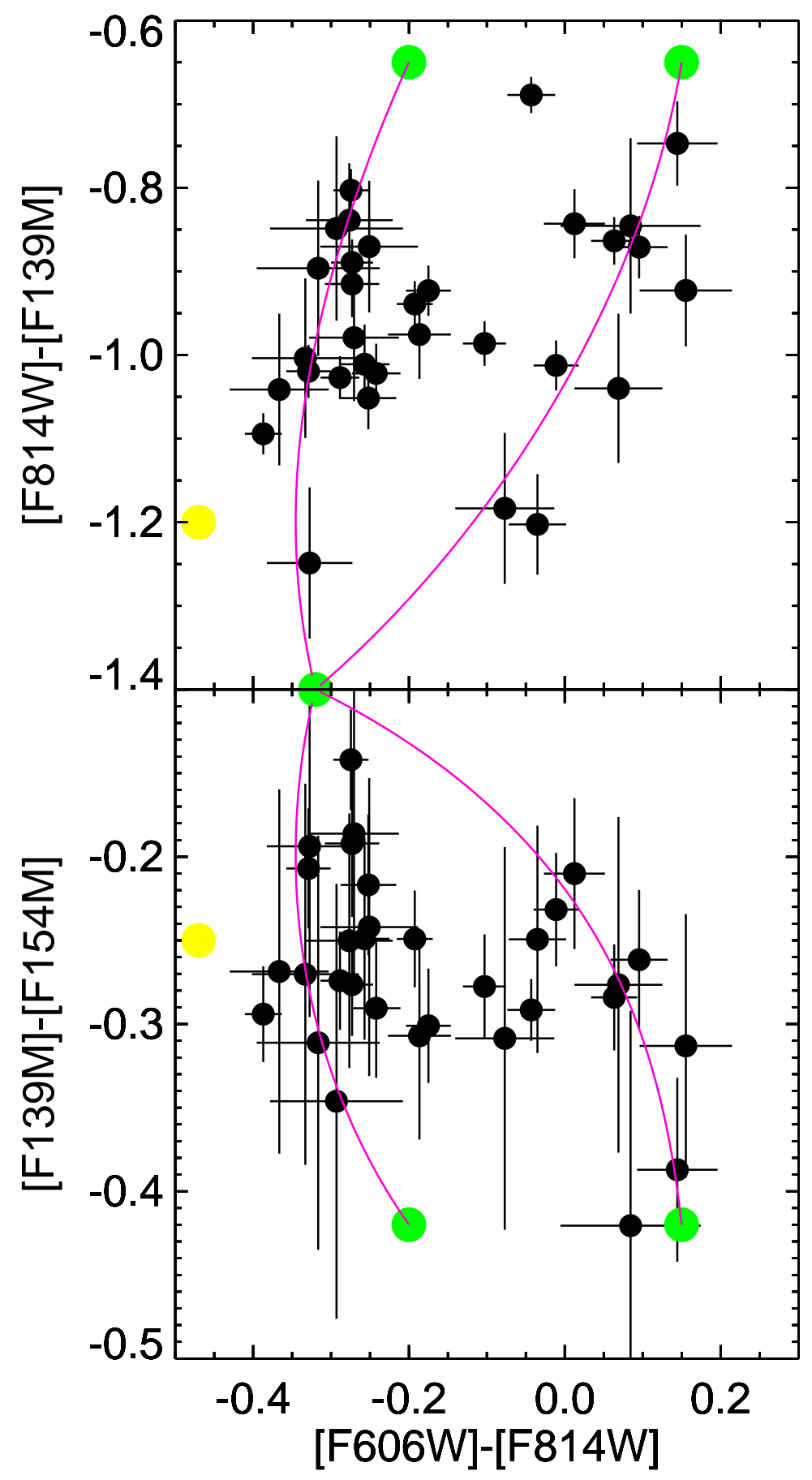

Fig. 7.- Three-color HST photometry of objects with perihelia inside of 30 AU from the H/WTSOSS survey. The filters are at 0.606, 0.8141 .39 , and $1.54 \mu \mathrm{m}$, corresponding roughly to $R, I, H$-continuum, and a filter sampling the $1.6 \mu \mathrm{m}$ water ice absorption feature. The yellow point shows the colors of the sun. The objects appear to bifurcate into two clumps, one with near-solar optical colors (the "neutral" clump) and one with significantly redder optical colors (the "red clump"). The neutral clump (on the left in [F606W]-[F814W] colors) shows a clear spread in the other colors, while the red clump shows a spread in all three colors. The color variation in both clumps can be described by a mixing line (purple lines) where the neutral and red clumps 
dance of low inclination, dynamically cold objects beyond about 41 AU (Brown 2001). Subsequent observations revealed that these objects shared a common red coloring (Trujillo \& Brown 2002). In the H/WTSOSS survey these objects do not fall along mixing lines, as the neutral and red clumps of centaurs do, but instead appear nearly uniform in color space (Fraser \& Brown 2011). These cold classical KBOs are also know to be unique in their lack of large bodies (Levison \& Stern 2001), their higher abundance of satellites (Noll et al. 2008), and their different size distribution (Fraser. Brown \& Schwamb 2010). And though our understanding of albedos in the Kuiper belt is still poor, preliminary results suggest that the cold classical KBOs also appear to have higher albedos than those of the remaining population (Brucker et al. 2009). All of these properties appear to signify a population with a different - and perhaps unique - formation location or history. Understanding the surface compositions of these objects will be a challenge given their distance and small sizes. In addition, these objects are dynamically stable, so we likely never get samples of this population as centaurs or comets.

\subsection{The diverse colors of the remainder of the Kuiper belt}

Other than the unique color properties of the centaurs and cold classical KBOs, the bulk of KBOs have no discernible pattern to their colors. This finding in itself is significant for understanding the causes of the colors of KBOs. This lack of a connection between color and any dynamical property, particularly with semi-major axis or perihelion/aphelion distance argues strongly that local heating, UV irradiation, and solar wind and cosmic ray bombardment (Cooper et al. 2003) cannot be responsible for the varying colors of the Kuiper belt. Local conditions appear to have no primary influence on the colors of KBOs. Furthermore, careful measurement of the colors of the separate components of binary KBOs has shown a tight correlation over the full range of Kuiper belt colors (Benecchi et al. 2009). The colors of two KBOs in orbit around each other are almost always nearly identical. This fact immediately rules out any of the stochastic process such as collisions for the causes of these Kuiper belt colors. Indeed, given the lack of correlation of color with local conditions, the nearly identical colors of binary KBOs argues that colors are simply primordial. If

binary KBOs were formed by early mutual capture in a quiescent disk (Goldreich, Lithwick \& Sari 2002), the two component would likely have formed in very similar locations. If, alternatively, binary KBOs were formed in an initial gravitational collapse (Nesvorný, Youdin \& Richardson 2010), the objects would of necessity have formed at the same location and of the same materials.

\subsection{The transition from KBOs to centaurs}

The manner in which primordial KBO surfaces evolve to become the color-bifurcated centaur population could provide important clues to the compositions of both surfaces. While it appears that a transition from a unimodal to bimodal color distribution must occur as objects move to lower perihelia, the actual evidence for change is, in fact, weak. The KBOs and centaurs with 
measured colors have very different ranges of sizes, and, as demonstrated above, large KBOs have their surfaces modified by the presence of volatiles and water ice. We thus must only compare likesized objects. In addition, the cold classical KBOs, with their unique surfaces, likely never enter the centaur population, so these should be excluded from the comparison. Finally, the KBOs, being more distant, are likely to have higher uncertainty in their color measurements.

When the centaurs are compared to the appropriate KBOs, little evidence of surface evolution can be found. In Figure 8 we compare well-measured optical colors of centaurs with absolute magnitudes between 6 and 9 to those of non-cold classical KBOs with the same range of absolute magnitudes. While colors of the Kuiper belt as a whole appear significantly different from those of the centaurs, the difference is much less apparent when we compare the correct samples. In fact, a Kolmogorov-Smirnov test of the two distributions cannot distinguish the two at greater than a $48 \%$ confidence limit. Using all of the available color data, we find no statistical evidence that any color evolution has occurred as objects move inward from the Kuiper belt to become centaurs. Fraser \& Brown (2011) come to the same conclusion from the optical and near-infrared photometry of the H/WTSOSS survey. In addition, the albedos of KBOs measured with Spitzer appear to be similarly distributed to those of the smaller KBOs (Stansberry et al. 2008).

While there is no evidence in the spectroscopic or photometric data for surface evolution in centaurs, in at least one way centaurs and KBOs are clearly different. A small number of centaurs is known to show cometary activity, at least sporadically (Jewitt 2009). To date, such activity has appeared confined to objects in the neutral clump, though the numbers remain small and the color measurements themselves could be compromised by the presence of a coma (Jewitt 2009). In addition, all but 2 of the 13 of the known active centaurs have perihelia inside of $10 \mathrm{AU}$. It has been speculated that outgassing could lead to surface modification and even to the bifurcated optical colors of the centaurs (Tegler et al. 2008a). Interestingly, an examination of the 16 centaurs observed in the H/WTSOSS survey shows no evidence for activity in any of them. Only 4 of the 16 have perihelia inside of $10 \mathrm{AU}$, though. Nonetheless, the colors bifurcation seen in this sample of currently inactive centaurs suggests that color bifurcation is not directly caused by centaur activity.

Robustly determining whether or not centaur surfaces are evolved is difficult. The numbers of centaurs available is small, the comparison KBOs are faint, and the expected distributions are unknown. It is possible that a complete understanding of this question will require large scale surveys like that of the currently-proposed Large Scale Synoptic Telescope (LSST) to find and characterize many more objects and acquire sufficient statistics to understand these populations.

\subsection{The causes of colors}

To date, only three hypotheses have been advanced to explain the colors of KBOs. The earliest hypotheses suggested randomized collisional excavation (Luu \& Jewitt 1996) or velocity dependent

impact resurfacing (Stern 2002), but the correlated colors of KBO binaries (Benecchi et al. 2009) 


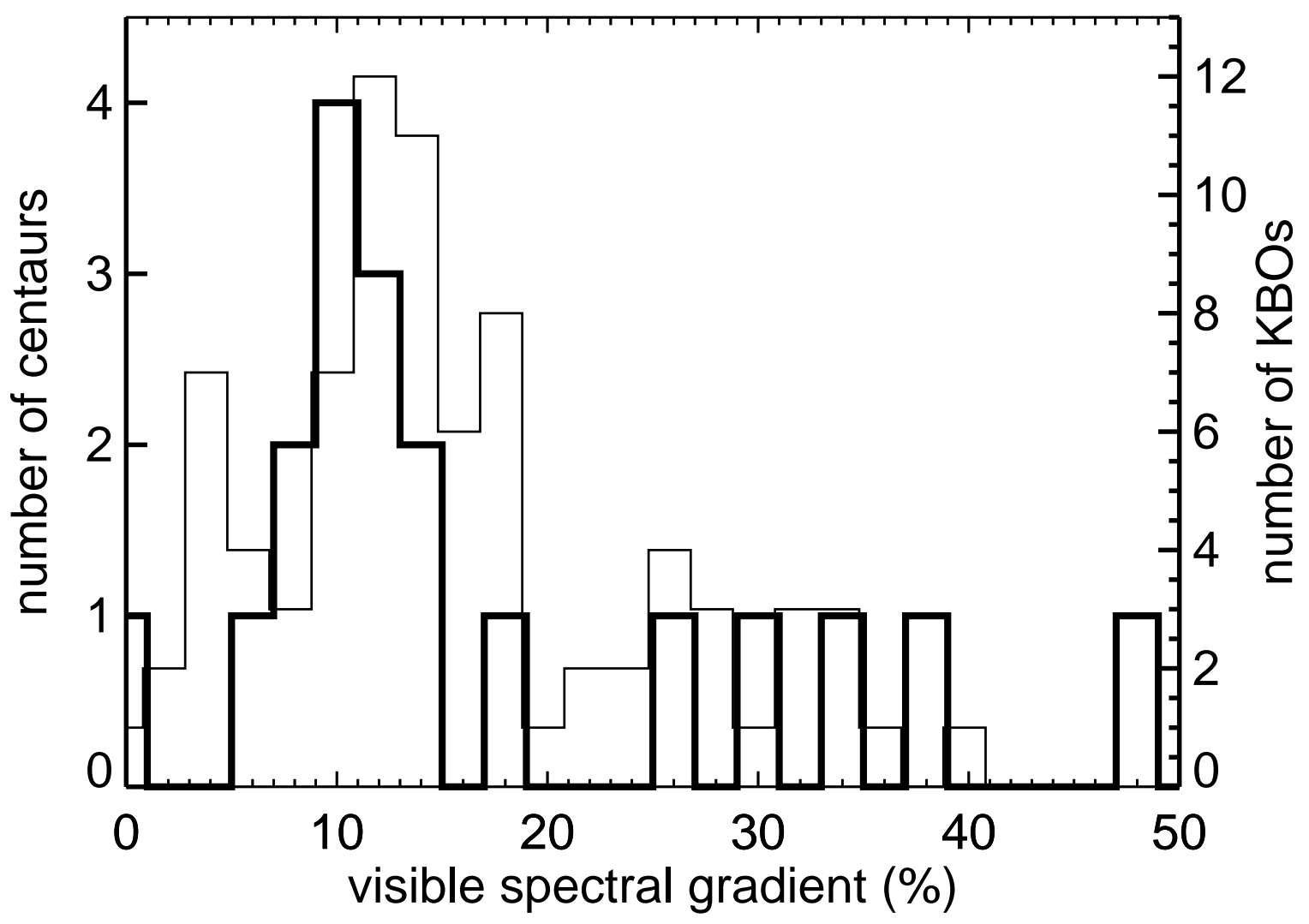

Fig. 8.- A comparison of the colors of (non-cold classical) KBOs (thin line) and centaurs (thick line) in the $6<H<9$ range. A K-S test cannot distinguish any significant difference between the distributions. While it is commonly assumed that the surfaces of KBOs evolve to have the color distribution of the centaur population, we find no statistical evidence that such evidence occurs. A similar conclusion can be drawn from the spectra of Figure 5 and from the three-color data of the H/WTSOSS survey. 
effectively rules out these or any other stochastic processes. Cooper et al. (2003) have suggested that the uniform red colors of the cold classical KBOs are caused by their existence in an irradiation minimum environment, with irradiation increasing interior due to increasing fluxes from solar energetic ions and increasing exterior by increased flux of energetic ions from the termination shock, but this hypothesis has no general explanation for the remaining colors, nor would it lead to the sizable observed population neutrally colored higher inclination objects in the same environment.

A new hypothesis has suggested that the colors of KBOs are set by early evaporation and irradiation of volatiles followed by dynamical mixing (Brown, Schaller \& Fraser 2011a). In this hypothesis, objects are formed outside of Neptune in the $\sim 15-30$ AU region in the early solar system out of a diverse and variable mix of materials. When the nebula disperses and objects are first exposed to sunlight, their surfaces heat, and volatiles are driven off. For widely variable starting compositions, the surface compositions of objects at the same distance from the sun will become nearly identical. Figure 9 shows a model from Brown. Schaller \& Fraser (2011a) which shows the distance at which different volatiles would be completely removed from the surface of an object as a function of size of the object. Regardless of the diversity of initial compositions, the surfaces of the objects will quickly have strong gradients in volatile composition. These volatiles are then irradiated and begin to develop the colors expected from their particular mix of remaining surface volatiles.

In this model, methanol is the most important coloring agent. Methanol is depleted on all surfaces inside of about $20 \mathrm{AU}$, and present on all of those exterior. While hydrocarbons are generally expected to carbonize and turn dark and neutral upon prolonged irradiation, laboratory experiments have suggested that irradiation of methanol to dosages expected on KBOs over the age of the solar system leads to bright red surfaces. In this hypothesis, then, the objects interior to the methanol line become the neutral population, while those outside become the red population. A solar system scale instability such as that envisioned by the Nice model then scatters the objects onto the orbits where they currently reside, mixing the neutral and red populations. The cold classical KBOs form in place (Batygin. Brown \& Betts 2012), and their unique colors form by the additional presence of ammonia on their surfaces. As KBOs scatter inward and become centaurs, their surfaces remain unchanged.

While the model of Brown, Schaller \& Fraser (2011a) is the only quantitative model to attempt to explain all of the current observations of colors of KBOs, it is clearly speculative. Much of the laboratory data required to trace the specific irradiation chemistry has not been performed, and the question of the evolution of the surfaces of the centaurs remains open. Extending this model to the Hubble Space Telescope survey of Fraser \& Brown (2011), which suggests mixing on the surfaces of KBOs, we would conclude that the irradiated volatiles are the neutral and red materials that are mixed in with the hydrated silicate-like material. No obvious explanation exists for the different amounts of mixing between the irradiated volatiles and the silicates. 


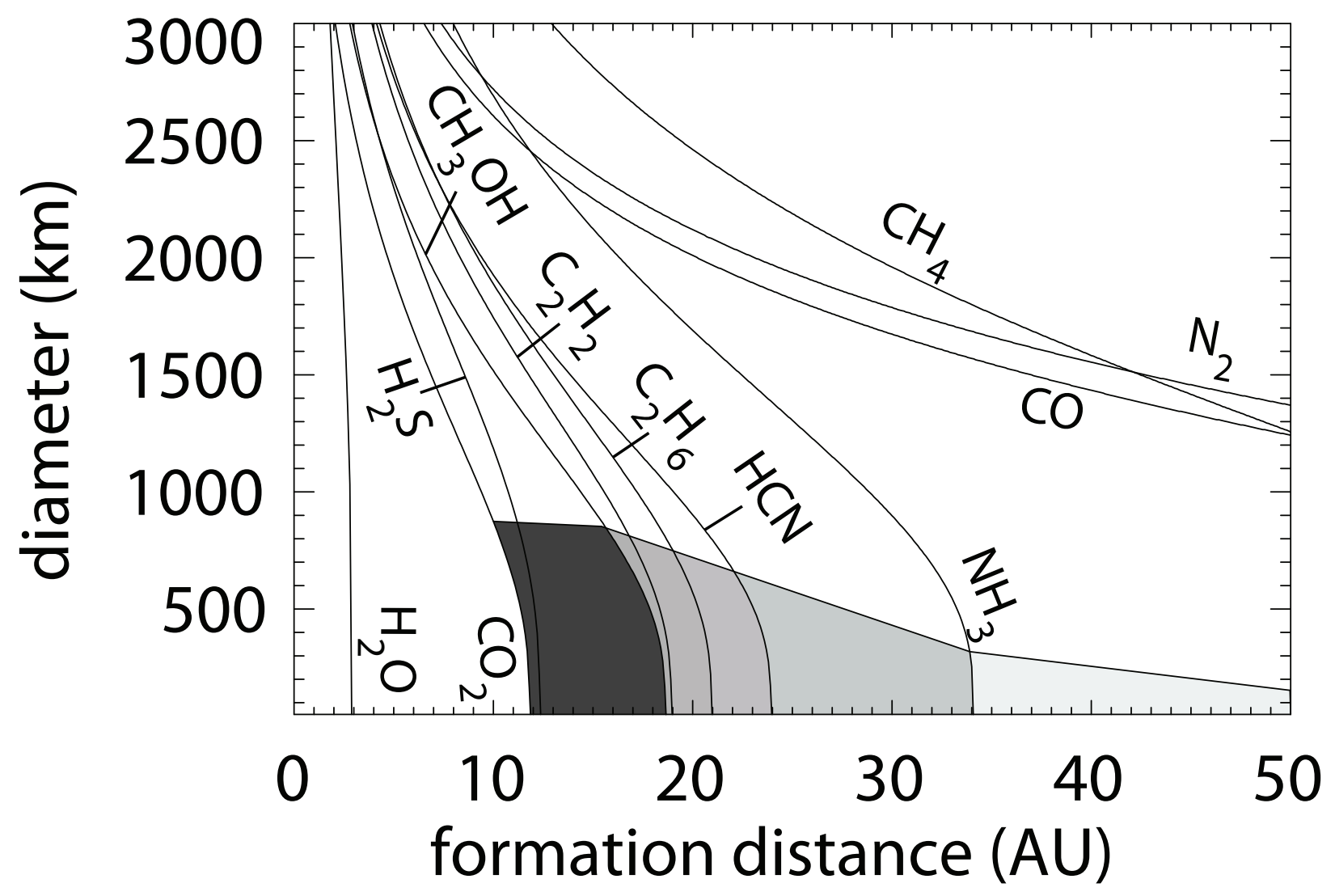

Fig. 9.- A hypothesis for the surface colors of small KBOs. In the early solar system KBOs form with a variety of composition, but as the nebula disappears and the sun begins to heat their surfaces, strong gradients in their surface compositions form. Leftward of each labeled line, each species would be fully depleted from the surface. KBOs formed interior to about 20 AU would only have $\mathrm{H}_{2} \mathrm{O}$ and $\mathrm{CO}_{2}$ on their surfaces, while KBOs which formed outside of $\sim 20 \mathrm{AU}$ would retain methanol on their surfaces. Irradiation of the methanol-free surfaces could lead to the dark neutral objects, while irradiation of methanol-containing surfaces could lead to the brighter red objects. Outside of $\sim 30 \mathrm{AU}$ objects can also retain $\mathrm{NH}_{3}$, which could perhaps explain some of the unique surface characteristics of the cold classical KBOs if they formed in situ. 


\section{Bulk composition}

While most work on the composition of the Kuiper belt has focused on the surface composition, all of the spectral and photometric results discussed probe an insignificantly small depth into the surface. To understand the true composition of KBOs requires an understanding of the bulk composition. Detailed measurement of the bulk composition is of course impossible, but one important proxy - the ice-to-rock fraction - is available for some KBOs. Measurement of the ice-to-rock ratio requires measurement of the density which, in turn, requires measurement of the mass and radius of the objects. While measurement of the size of an object is possible through multiple means (to date far infrared radiometry from the Spitzer Space Telescope has been the dominant method), a measurement of the mass is only possible if the object has a satellite whose orbit is known.

KBOs were expected to be a relatively homogeneous group. They all are thought to have grown gradually through accretion, sampling similar regions of the solar nebula, so their compositions should have been nearly identical. Indeed, when Pluto was the only known large Kuiper belt object, its density of $\sim 2 \mathrm{~g} \mathrm{~cm}^{-3}$ was taken to indicate a $\sim 30-70$ ice-rock mix in the outer solar nebula as a whole (McKinnon \& Mueller 1988).

One of the biggest outer solar system surprises of the past few years, therefore, has been the discovery that the ice fraction measured in KBOs varies from essentially 0 to 1 (Figure 10). Objects have been found with densities significantly less than $1 \mathrm{~g} \mathrm{~cm}^{-3}$ (Mueller et al. 2009; Benecchi et al. 2010) indicating both a near-unity ice fraction and significant porosity, while other objects have been found with densities of nearly pure rock (Fraser \& Brown 2010).

Examination of the measured densities diameter reveals that the density measurements have extremely large uncertainties. The large error bars are a function of the uncertainty in the measured sizes and the 3-times-higher uncertainties in the associated volumes. Detailed understanding of the trends and causes of ice-rock fractions in the Kuiper belt clearly requires significantly higher quality size measurements.

Even with these large uncertainties, however, two trends are apparent. First, there is a general trend for an increase in density as a function of size. While an object with a fixed ice fraction will undergo a density increase with increased size owing to the small density increase that occurs due to the change of ice to higher density phase as pressure is increased, the general trend of increased density with size seen in the Kuiper belt is significantly larger than expected unless the rock fraction itself is increasing in larger objects (Fig. 10).

The second general trend is the difference in densities between objects which exclusively have small satellites and those which have larger satellites. The objects which exclusively have small satellites - Haumea, Quaoar, and Eris - have been hypothesized to have undergone giant impacts

which led to these satellite (Brown et al. 2006). Interestingly, these objects have higher densities than every other measured object.

Such a wide range of ice-rock ratios is astounding. No dynamical evidence exists that the large 


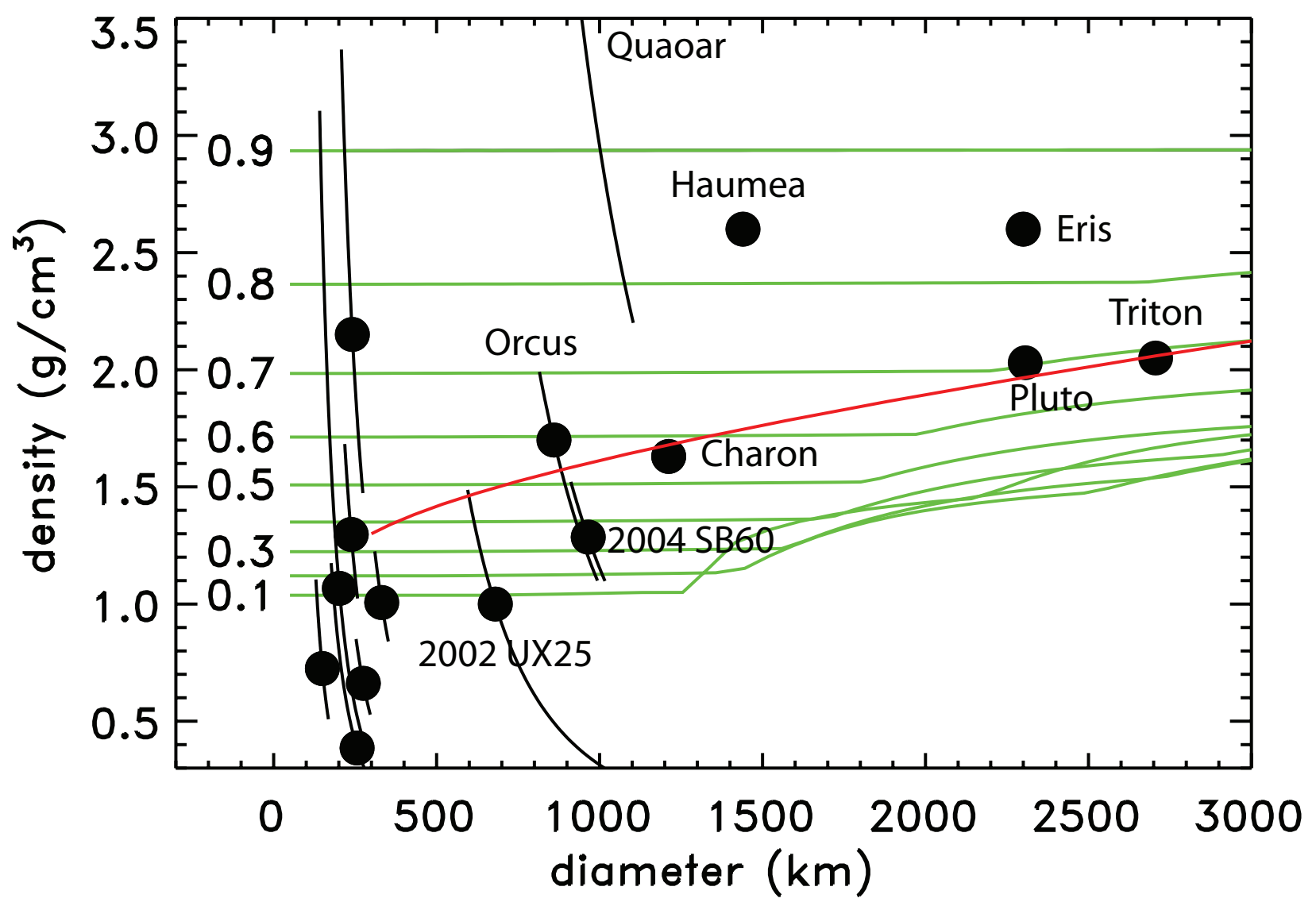

Fig. 10.- Measured densities of KBOs. In all cases the uncertainty in the size dominates the density measurement, so error bars follow lines of constant mass with varying diameter. The green lines show the density as a function of size for different rock fractions for fully differentiated body; the ice phase change at higher pressures causes ice to get denser. The red line shows the maximum density increase that could be expected from a simple model where small amounts of ice are preferentially removed in the collisions required to build larger bodies from smaller ones. Data for sizes and densities come from Rabinowitz et al. (2006); Brown \& Schaller (2007); Mueller et al. (2009); Brown et al. (2010); Fraser \& Brown (2010); Benecchi et al. (2010). 
KBOs came from dramatically different regions of the solar nebula, and no reasonable formation mechanism appears capable of delivering such variability among large accreted objects. We suggest three scenarios for explaining the extreme variability of the bulk composition of KBOs below, but we do not find any of the explanations completely satisfying.

\subsection{Single collision densification}

The extreme densities of all of the KBOs which exclusively have small satellites immediately suggests a connection between the satellite-forming giant impact and the high density. Indeed, in the case of Haumea, the creation of an icy collisional family dynamically surrounding the object demonstrates that these impacts do indeed remove ice from a differentiated mantle. It appears reasonable to suggest that the three known objects with the highest densities all achieved these densities through single catastrophic collisions which removed substantial amounts of overlying ice in a process reminiscent of that envisioned for Mercury.

Unfortunately, the types of collisions that would be required are infeasible in the solar system. Using scalings from numerical simulations of Stewart \& Leinhardt (2009), we find that to remove enough ice mass to create an object with the $\sim 2.6 \mathrm{~g} \mathrm{~cm}^{-3}$ of Eris from an object that initially had the $\sim 2.0 \mathrm{~g} \mathrm{~cm}^{-3}$ of Pluto requires hitting Triton with a $\sim 500 \mathrm{~km} \mathrm{KBO}$ traveling $40 \mathrm{~km} \mathrm{~s}^{-1}$ !

Though the connection between high densities and small satellites is striking, we can envision no physical mechanism by which the two can be related.

\subsection{Water ice loss during accretion}

While single impacts cannot lead to significant ice loss, multiple impacts during accretion can. The red line in Figure 10 shows, for example, a scenario where large bodies are preferentially grown by accreting other like-sized bodies. For two bodies which collide at their mutual escape velocities, as such bodies would, the Stewart \& Leinhardt (2009) scalings suggest that $\sim 10 \%$ of the total mass will be removed from the system. If for differentiated objects the ice is exclusively removed, these objects will grow in density as they grow in size.

The model shown here then represent the maximum density growth that could happen from an assumed starting size and starting density $\left(300 \mathrm{~km}, 1.3 \mathrm{~g} \mathrm{~cm}^{-3}\right)$ for differentiated objects which grow to become the larger dwarf planets. While a general increase such as that seen through Charon, Pluto, and Triton can perhaps be explained, the three very high density objects are well away from this line. To create Quaoar, for example, would require starting with $300 \mathrm{~km}$ differentiated objects with initial densities of $1.9 \mathrm{~g} \mathrm{~cm}^{-3}$, which would then be inconsistent with the other observed densities.

While accretional loss of water ice may play some role in the general trend of increasing 
densities with size, that process clearly cannot explain all of the observations.

\subsection{Extreme inhomogeneity in the disk}

One of the only alternatives to invoking collisions as a means to modify densities is to suggest that the densities are primordial. The general trend of densities increasing with size could be caused by an increase in the ice-to-rock ratio as a function of heliocentric distance, combined by a tendency of the largest objects to form closer to the sun where formation timescales are shorter and larger objects can grow. However, this scenario would also suggest the existence of a population of smaller objects which also formed at these closer distances and should therefore also have the high densities of the largest objects. No such population appears.

The alternative is that the ice-to-rock ratio did not vary smoothly with distance, but rather the disk was chemically inhomogeneous. It is difficult to reconcile this possibility with the assumption that the large objects were accreted out of large numbers of small ones sampling multiple portions of the disk.

None of the three proposed scenarios provides a satisfactory explanation for the trends and variability seen in densities. The study of the bulk composition of KBOs is, however, in its infancy. Radii of KBOs are still poorly determined, and the number of objects with measured satellite orbits remains small. We anticipate that continued work in this areas will eventually yield the insights that will allow the bulk compositions of these objects to be understood.

\section{Implications, speculations, and open questions}

We have attempted to discern general trends and formulate overlying principles for understanding the compositions of the objects in the Kuiper belt. We are at a stage in our understanding of the Kuiper belt that, for the first time, we have at least a first-order understanding of the compositions of the surfaces of KBOs and valid working hypotheses for understanding these compositions. A comparable understanding of the bulk composition is - however - far away.

Using all of the spectroscopic and photometric data as well as the current best understanding of the physics and chemistry of these bodies, we conceptually divide Kuiper belt surfaces into major groups, with differences being caused by size, formation location, and history. The six broad types of KBO surfaces are as follow:

- Volatile rich (Triton, Eris, Pluto): objects large enough to retain significant reservoirs of $\mathrm{N}_{2}$, $\mathrm{CH}_{4}$, and $\mathrm{CO}$. Their spectra are dominated by $\mathrm{CH}_{4}$ absorption, but their surfaces are likely dominated by $\mathrm{N}_{2}$ with $\mathrm{CH}_{4}$ a moderate contributor. 
- Volatile transition (Makemake, Quaoar, 2007 OR10): objects which are on the verge of losing their volatiles but still contain (at least) $\mathrm{CH}_{4}$. With $\mathrm{CH}_{4}$ the dominant molecule on the surface, radiation processing occurs much more quickly, so ethane and other methane irradiation products are present on the surface.

- Water ice plus ammonia rich surfaces (Charon, Orcus, Quaoar, 2007 OR10, AZ84, other): Slightly smaller $(\sim 500 \mathrm{~km}<D<\sim 1200 \mathrm{~km})$ objects on which volatiles have been mostly or fully depleted and the spectra are dominated by significant crystalline water ice absorption. Where high $\mathrm{S} / \mathrm{N}$ is available and methane doesn't hide the spectral signature, we expect that all of these will show the presence of ammonia. We suggest that these are due to water ice flowing on the surface after volatile irradiation has otherwise set the surface color and composition.

- Neutral surfaces of small objects: Objects which are too small to retain volatiles or to have had water flows and formed inside $\sim 20$ AU will have their surfaces depleted in all major ices except $\mathrm{H}_{2} \mathrm{O}$ and $\mathrm{CO}_{2}$. Irradiation will then cause dark neutrally colored surfaces to develop. These objects show a range of optical-near IR colors based on the amount of silicate mixed with the irradiated ices. No hypothesis has been suggested for why objects differ in their surface rock-ice ratio.

- Red surfaces of small objects: Small objects which formed outside of $\sim 20$ AU would have been able to retain $\mathrm{CH}_{3} \mathrm{OH}$ on their surface. Upon irradiation these surfaces would turn red and retain moderate albedos. These objects show a range of optical and optical-near IR colors based on the amount of silicate mixed with the irradiated ices.

- Pure water ice (Haumea and its family and satellites): pure water ice surfaces form when the nearly-pristine water ice mantle of a differentiated KBO is exposed in an impact.

While these six classes of Kuiper belt surfaces provide an overall framework for understanding the the composition of KBO surfaces, there are open questions about multiple aspects of this framework that must be answered before we can be certain that our first-order understanding of these surfaces is correct. In addition, some overall questions still remain. We summarize some of the most important questions to be answered by future research:

- Is water ice on medium-sized KBOs a sign of previous water flow on the surface? Evidence that this hypothesis is true would include the presence of ammonia on every medium-sized objects on which substantial water ice absorption is present. Images of Charon from the New Horizon spacecraft may also shed light on this question.

- Is the sporadic methanol seen on small objects a transient sign of a recent impact? Direct evidence for this hypothesis is difficult without resolved imaging, for which there are no future plans. However, rotationally revolved spectroscopy could help begin to answer this question. 
Such spectroscopy will require the next generation of large telescopes, but rotationally resolved color measurement may provide a currently feasible initial step.

- Do KBO surfaces become modified as they enter the centaur region? Extremely high precision color measurements of large numbers of higher perihelion non-cold classical KBOs could answer this question. Such precise measurements are possible with current telescopes. Understanding the conditions that lead to centaur activity and that effect of activity on the surface - if any - will also be important.

- Can the volatile loss hypothesis for the colors of KBOs be supported by new laboratory data? The hypothesis suggests specific chemical combinations that give the colors seen in the Kuiper belt; irradiation of these combinations would show if the predictions are valid.

- Are silicates visible at the surface? Reliably reproducible results on the presence of aqueously altered silicates would be strong evidence. Midinfrared spectroscopy is difficult but should eventually prove feasible. The silicate-colored material identified in the mixtures from the purely photometric H/WTSOSS surface could perhaps be isolated spectroscopically with carefully targeted measurements.

- What causes the extreme bulk compositional variability of the Kuiper belt? Three hypotheses have been suggested, but none of them is satisfactory. Significant more work is needed in this area.

The last decade has seen studies of the composition of the Kuiper belt blossom from simple cataloging to the development of actual conceptual frameworks which can explain many of the observed properties. Much work remains, however, to both verify and extend this framework, and to use these distant bodies to continue to provide new insights into the formation and evolution of the solar system.

The preparation of this review has been supported by grant NNX09AB49G from the NASA Planetary Astronomy program.

\section{REFERENCES}

Alvarez-Candal A, Fornasier S, Barucci MA, de Bergh C, Merlin F. 2008, Visible spectroscopy of the new ESO large program on trans-Neptunian objects and Centaurs. Part 1. AESA 487:741-748

Barkume KM, Brown ME, Schaller EL. 2006, Water Ice on the Satellite of Kuiper Belt Object 2003 EL61. ApJ 640:L87-L89

Barkume KM, Brown ME, Schaller EL. 2008, Near-Infrared Spectra of Centaurs and Kuiper Belt Objects. AJ 135:55-67 
Barucci MA, Alvarez-Candal A, Merlin F, Belskaya IN, de Bergh C, et al. 2011, New insights on ices in Centaur and Transneptunian populations. Icarus 214:297-307

Barucci MA, Brown ME, Emery JP, Merlin F. 2008a. Composition and Surface Properties of Transneptunian Objects and Centaurs, In The Solar System Beyond Neptune, eds. MA Barucci, H Boehnhardt, DP Cruikshank, A Morbidelli. 143-160

Barucci MA, Cruikshank DP, Dotto E, Merlin F, Poulet F, et al. 2005, Is Sedna another Triton? AESA 439:L1-L4

Barucci MA, Merlin F, Dotto E, Doressoundiram A, de Bergh C. 2006, TNO surface ices. Observations of the TNO 55638 (2002 VE95). A\&A 455:725-730

Barucci MA, Merlin F, Guilbert A, de Bergh C, Alvarez-Candal A, et al. 2008b, Surface composition and temperature of the TNO Orcus. A\&SA 479:L13-L16

Barucci MA, Morea Dalle Ore C, Alvarez-Candal A, de Bergh C, Merlin F, et al. 2010, (90377) Sedna: Investigation of Surface Compositional Variation. AJ 140:2095-2100

Batygin K, Brown ME, Betts H. 2012, Instability-driven Dynamical Evolution Model of a Primordially Five-planet Outer Solar System. ApJ 744:L3

Benecchi SD, Noll KS, Grundy WM, Buie MW, Stephens DC, Levison HF. 2009, The correlated colors of transneptunian binaries. Icarus 200:292-303

Benecchi SD, Noll KS, Grundy WM, Levison HF. 2010, (47171) 1999 TC36, A transneptunian triple. Icarus 207:978-991

Bennett CJ, Jamieson CS, Osamura Y, Kaiser RI. 2006, Laboratory Studies on the Irradiation of Methane in Interstellar, Cometary, and Solar System Ices. ApJ 653:792-811

Bernstein G, Khushalani B. 2000, Orbit Fitting and Uncertainties for Kuiper Belt Objects. AJ 120:3323-3332

Brown ME. 2001, The Inclination Distribution of the Kuiper Belt. AJ 121:2804-2814

Brown ME. 2002, Pluto and Charon: Formation, Seasons, Composition. Annual Review of Earth and Planetary Sciences 30:307-345

Brown ME. 2008. The Largest Kuiper Belt Objects, In The Solar System Beyond Neptune, eds. MA Barucci, H Boehnhardt, DP Cruikshank, A Morbidelli. 335-344

Brown ME, Barkume KM, Blake GA, Schaller EL, Rabinowitz DL, et al. 2007a, Methane and Ethane on the Bright Kuiper Belt Object 2005 FY9. AJ 133:284-289

Brown ME, Barkume KM, Ragozzine D, Schaller EL. 2007b, A collisional family of icy objects in the Kuiper belt. Nature 446:294-296 
Brown ME, Bouchez AH, Rabinowitz D, Sari R, Trujillo CA, et al. 2005, Keck Observatory Laser Guide Star Adaptive Optics Discovery and Characterization of a Satellite to the Large Kuiper Belt Object 2003 EL61. ApJ 632:L45-L48

Brown ME, Burgasser AJ, Fraser WC. 2011, The Surface Composition of Large Kuiper Belt Object 2007 OR10. ApJ 738:L26+

Brown ME, Calvin WM. 2000, Evidence for Crystalline Water and Ammonia Ices on Pluto's Satellite Charon. Science 287:107-109

Brown ME, Ragozzine D, Stansberry J, Fraser WC. 2010, The Size, Density, and Formation of the Orcus-Vanth System in the Kuiper Belt. AJ 139:2700-2705

Brown ME, Schaller EL. 2007, The Mass of Dwarf Planet Eris. Science 316:1585-

Brown ME, Schaller EL, Fraser WC. 2011a, A Hypothesis for the Color Diversity of the Kuiper Belt. ApJ 739:L60

Brown ME, Schaller EL, Fraser WC. 2011b, Ices on medium sized Kuiper belt objects. Astron. J., submitted

Brown ME, Trujillo CA, Rabinowitz DL. 2005, Discovery of a Planetary-sized Object in the Scattered Kuiper Belt. ApJ 635:L97-L100

Brown ME, van Dam MA, Bouchez AH, Le Mignant D, Campbell RD, et al. 2006, Satellites of the Largest Kuiper Belt Objects. ApJ 639:L43-L46

Brown R, Cruikshank D, Ververka J, Helfenstein P, Eluszkiewicz J. 1995. Surface Composition and Photometric Properties of Triton, In Neptune and Triton, ed. DP Cruikshank

Brucker MJ, Grundy WM, Stansberry JA, Spencer JR, Sheppard SS, et al. 2009, High albedos of low inclination Classical Kuiper belt objects. Icarus 201:284-294

Brunetto R, Barucci MA, Dotto E, Strazzulla G. 2006, Ion Irradiation of Frozen Methanol, Methane, and Benzene: Linking to the Colors of Centaurs and Trans-Neptunian Objects. ApJ 644:646650

Cook JC, Desch SJ, Roush TL, Trujillo CA, Geballe TR. 2007, Near-Infrared Spectroscopy of Charon: Possible Evidence for Cryovolcanism on Kuiper Belt Objects. ApJ 663:1406-1419

Cooper JF, Christian ER, Richardson JD, Wang C. 2003, Proton Irradiation of Centaur, Kuiper Belt, and Oort Cloud Objects at Plasma to Cosmic Ray Energy. Earth Moon and Planets 92:261-277

Cruikshank DP, Roush TL, Bartholomew MJ, Geballe TR, Pendleton YJ, et al. 1998, The Composition of Centaur 5145 Pholus. Icarus 135:389-407 
Cruikshank DP, Roush TL, Moore JM, Sykes M, Owen TC, et al. 1997. The Surfaces of Pluto and Charon, In Pluto and Charon, ed. Stern, S. A. \& Tholen, D. J. 221-+

Cruikshank DP, Roush TL, Owen TC, Geballe TR, de Bergh C, et al. 1993, Ices on the surface of Triton. Science 261:742-745

Davies JK, Sykes MV, Cruikshank DP. 1993, Near-infrared photometry and spectroscopy of the unusual minor planet 5145 Pholus (1992AD). Icarus 102:166-169

de Bergh C, Boehnhardt H, Barucci MA, Lazzarin M, Fornasier S, et al. 2004, Aqueous altered silicates at the surface of two Plutinos? A\&\& 416:791-798

Delsanti A, Merlin F, Guilbert-Lepoutre A, Bauer J, Yang B, Meech KJ. 2010, Methane, ammonia, and their irradiation products at the surface of an intermediate-size KBO?. A portrait of Plutino (90482) Orcus. A\&A 520:A40+

DeMeo FE, Dumas C, de Bergh C, Protopapa S, Cruikshank DP, et al. 2010, A search for ethane on Pluto and Triton. Icarus 208:412-424

Doressoundiram A, Boehnhardt H, Tegler SC, Trujillo C. 2008. Color Properties and Trends of the Transneptunian Objects, In The Solar System Beyond Neptune, ed. Barucci, M. A., Boehnhardt, H., Cruikshank, D. P., Morbidelli, A., \& Dotson, R. 91-104

Douté S, Schmitt B, Quirico E, Owen TC, Cruikshank DP, et al. 1999, Evidence for Methane Segregation at the Surface of Pluto. Icarus 142:421-444

Dumas C, Carry B, Hestroffer D, Merlin F. 2011, High-contrast observations of (136108) Haumea. A crystalline water-ice multiple system. A\&A 528:A105+

Eluszkiewicz J, Cady-Pereira K, Brown ME, Stansberry JA. 2007, Interpretation of the near-IR spectra of the Kuiper Belt Object (136472) 2005 FY9. Journal of Geophysical Research (Planets) 112:E06003

Emery JP, Cruikshank DP, van Cleve J. 2006, Thermal emission spectroscopy (5.2 $38 \mu \mathrm{m})$ of three Trojan asteroids with the Spitzer Space Telescope: Detection of fine-grained silicates. Icarus 182:496-512

Fornasier S, Barucci MA, de Bergh C, Alvarez-Candal A, DeMeo F, et al. 2009, Visible spectroscopy of the new ESO large programme on trans-Neptunian objects and Centaurs: final results. A\&A 508:457-465

Fornasier S, Doressoundiram A, Tozzi GP, Barucci MA, Boehnhardt H, et al. 2004, ESO Large Program on physical studies of Trans-Neptunian objects and Centaurs: Final results of the visible spectrophotometric observations. A\&A 421:353-363 
Fraser WC, Brown ME. 2009, NICMOS Photometry of the Unusual Dwarf Planet Haumea and its Satellites. ApJ 695:L1-L3

Fraser WC, Brown ME. 2010, Quaoar: A Rock in the Kuiper Belt. ApJ 714:1547-1550

Fraser WC, Brown ME. 2011, The Hubble/WFC3 Test of Surfaces in Outer Solar System. Astron. J., submitted

Fraser WC, Brown ME, Schwamb ME. 2010, The luminosity function of the hot and cold Kuiper belt populations. Icarus 210:944-955

Goldreich P, Lithwick Y, Sari R. 2002, Formation of Kuiper-belt binaries by dynamical friction and three-body encounters. Nature 420:643-646

Guilbert A, Alvarez-Candal A, Merlin F, Barucci MA, Dumas C, et al. 2009, ESO-Large Program on TNOs: Near-infrared spectroscopy with SINFONI. Icarus 201:272-283

Hudson RL, Palumbo ME, Strazzulla G, Moore MH, Cooper JF, Sturner SJ. 2008. Laboratory Studies of the Chemistry of Transneptunian Object Surface Materials, In The Solar System Beyond Neptune, ed. Barucci, M. A., Boehnhardt, H., Cruikshank, D. P., Morbidelli, A., \& Dotson, R. 507-523

Jewitt D. 2009, The Active Centaurs. AJ 137:4296-4312

Jewitt DC, Luu J. 2004, Crystalline water ice on the Kuiper belt object (50000) Quaoar. Nature 432:731-733

Lazzarin M, Barucci MA, Boehnhardt H, Tozzi GP, de Bergh C, Dotto E. 2003, ESO Large Programme on Physical Studies of Trans-Neptunian Objects and Centaurs: Visible Spectroscopy. AJ 125:1554-1558

Levison HF, Morbidelli A, Vokrouhlický D, Bottke WF. 2008, On a Scattered-Disk Origin for the 2003 EL61 Collisional Family - An Example of the Importance of Collisions on the Dynamics of Small Bodies. AJ 136:1079-1088

Levison HF, Stern SA. 2001, On the Size Dependence of the Inclination Distribution of the Main Kuiper Belt. AJ 121:1730-1735

Licandro J, Pinilla-Alonso N, Pedani M, Oliva E, Tozzi GP, Grundy WM. 2006, The methane ice rich surface of large TNO 2005 FY9: a Pluto-twin in the trans-neptunian belt? $A \& A$ 445:L35-L38

Luu J, Jewitt D. 1996, Color Diversity Among the Centaurs and Kuiper Belt Objects. AJ 112:2310$+$

McKinnon WB, Mueller S. 1988, Pluto's structure and composition suggest origin in the solar, not a planetary, nebula. Nature 335:240-243 
Merlin F, Barucci MA, de Bergh C, DeMeo FE, Alvarez-Candal A, et al. 2010a, Chemical and physical properties of the variegated Pluto and Charon surfaces. Icarus 210:930-943

Merlin F, Barucci MA, de Bergh C, Fornasier S, Doressoundiram A, et al. 2010b, Surface composition and physical properties of several trans-neptunian objects from the Hapke scattering theory and Shkuratov model. Icarus 208:945-954

Morbidelli A, Brown ME. 2005. Comets II, ed. M.C. Festou, H.U. Keller, H.A. Weaver (U. Arizona Press: 2005)

Morbidelli A, Levison HF, Gomes R. 2008. The Dynamical Structure of the Kuiper Belt and Its Primordial Origin, In The Solar System Beyond Neptune, ed. Barucci, M. A., Boehnhardt, H., Cruikshank, D. P., Morbidelli, A., \& Dotson, R. 275-292

Mueller M, Spencer J, Stansberry J, Grundy W. 2009. In AAS/Division for Planetary Sciences Meeting Abstracts \#41, vol. 41 of AAS/Division for Planetary Sciences Meeting Abstracts

Nesvorný D, Youdin AN, Richardson DC. 2010, Formation of Kuiper Belt Binaries by Gravitational Collapse. AJ 140:785-793

Noll KS, Grundy WM, Stephens DC, Levison HF, Kern SD. 2008, Evidence for two populations of classical transneptunian objects: The strong inclination dependence of classical binaries. Icarus 194:758-768

Owen TC, Roush TL, Cruikshank DP, Elliot JL, Young LA, et al. 1993, Surface ices and the atmospheric composition of Pluto. Science 261:745-748

Rabinowitz DL, Barkume K, Brown ME, Roe H, Schwartz M, et al. 2006, Photometric Observations Constraining the Size, Shape, and Albedo of 2003 EL61, a Rapidly Rotating, Pluto-sized Object in the Kuiper Belt. ApJ 639:1238-1251

Rabinowitz DL, Schaefer BE, Schaefer M, Tourtellotte SW. 2008, The Youthful Appearance of the 2003 EL61 Collisional Family. AJ 136:1502-1509

Ragozzine D, Brown ME. 2007, Candidate Members and Age Estimate of the Family of Kuiper Belt Object 2003 EL61. AJ 134:2160-2167

Ragozzine D, Brown ME. 2009, Orbits and Masses of the Satellites of the Dwarf Planet Haumea (2003 EL61). AJ 137:4766-4776

Schaller EL, Brown ME. 2007a, Detection of Methane on Kuiper Belt Object (50000) Quaoar. ApJ 670:L49-L51

Schaller EL, Brown ME. 2007b, Volatile Loss and Retention on Kuiper Belt Objects. ApJ 659:L61L64 
Schwamb ME, Brown ME, Rabinowitz DL, Ragozzine D. 2010, Properties of the Distant Kuiper Belt: Results from the Palomar Distant Solar System Survey. ApJ 720:1691-1707

Sheppard SS, Lacerda P, Ortiz JL. 2008. Photometric Lightcurves of Transneptunian Objects and Centaurs: Rotations, Shapes, and Densities, In The Solar System Beyond Neptune, ed. Barucci, M. A., Boehnhardt, H., Cruikshank, D. P., Morbidelli, A., \& Dotson, R. 129-142

Stansberry J, Grundy W, Brown M, Cruikshank D, Spencer J, et al. 2008. Physical Properties of Kuiper Belt and Centaur Objects: Constraints from the Spitzer Space Telescope, In The Solar System Beyond Neptune, eds. MA Barucci, H Boehnhardt, DP Cruikshank, A Morbidelli. $161-179$

Stern SA. 2002, Evidence for a Collisional Mechanism Affecting Kuiper Belt Object Colors. AJ 124:2297-2299

Stewart ST, Leinhardt ZM. 2009, Velocity-Dependent Catastrophic Disruption Criteria for Planetesimals. ApJ 691:L133-L137

Tegler SC, Bauer JM, Romanishin W, Peixinho N. 2008a. Colors of Centaurs, In The Solar System Beyond Neptune, ed. Barucci, M. A., Boehnhardt, H., Cruikshank, D. P., Morbidelli, A., \& Dotson, R. 105-114

Tegler SC, Cornelison DM, Grundy WM, Romanishin W, Abernathy MR, et al. 2010, Methane and Nitrogen Abundances on Pluto and Eris. ApJ 725:1296-1305

Tegler SC, Grundy WM, Vilas F, Romanishin W, Cornelison DM, Consolmagno GJ. 2008b, Evidence of $\mathrm{N}_{2}$-ice on the surface of the icy dwarf Planet 136472 (2005 FY9). Icarus 195:844-850

Trujillo CA, Brown ME. 2002, A Correlation between Inclination and Color in the Classical Kuiper Belt. ApJ 566:L125-L128

Trujillo CA, Brown ME. 2003, The Caltech Wide Area Sky Survey. Earth Moon and Planets 92:99-112

Trujillo CA, Brown ME, Barkume KM, Schaller EL, Rabinowitz DL. 2007, The Surface of 2003 EL61 in the Near-Infrared. ApJ 655:1172-1178

Tryka KA, Brown RH, Anicich V. 1995, Near-infrared absorption coefficients of solid nitrogen as a function of temperature. Icarus 116:409-414 